\title{
Distributed Control for Cooperative Manipulation with Event-Triggered Communication
}

\author{
Pablo Budde gen. Dohmann Student Member, IEEE,Sandra Hirche Senior Member, IEEE
}

\begin{abstract}
Cooperative manipulation tasks can be divided into the sub-tasks of object trajectory tracking and grasp maintenance. Both sub-tasks typically pose individual requirements on the underlying control objective in terms of accuracy and robustness to disturbances. We propose a novel distributed impedance control scheme, resulting in a flexible control design in order to meet the individual - potentially conflicting - impedance goals of the respective sub-tasks. In order to achieve a more efficient use of the communication resource, we propose an eventtriggered strategy for the communication between the robotic agents. Simulations and experimental results show that with the proposed communication strategy the number of transmissions between agents can be significantly reduced, while maintaining the flexibility introduced by the distributed controller.
\end{abstract}

Index Terms-Networked Robots, Distributed Robot Systems, Multi-Robot Systems, Cooperative Manipulators

\section{INTRODUCTION}

$\mathbf{R}$ ECENT successes in the development of lightweight robots facilitate the ubiquitous deployment of costefficient, safe, and easy to use robots for everyday tasks. However, due to the inherited reduced payload compared to classical industrial robots it becomes unfeasible to manipulate heavy and bulky objects. As a consequence, cooperative manipulation in multi-robot systems has attracted a lot of attention recently [1]-[3]. Additionally, increasing the number of robots naturally comes with increasing degrees of freedom, which can be exploited for secondary tasks as obstacle avoidance or increasing manipulability [4]. Control strategies for multi-robot systems are typically distinguished into three approaches [5]. Centralized approaches, where the whole system is controlled from a single point, are highly performant. However, due to the requirement of a central unit for control, those come with high communication requirements and vulnerability against single-point failures, making them less robust and prone to attacks. In contrast, in decentralized approaches every agent is locally controlled without using information from its neighbors, which in general results in low performance. The performance of decentralized approaches can be increased by allowing for a certain degree of communication between agents, resulting in distributed control. However, distributed (as well as centralized) approaches require communication between the individual agents, preferably

This work was supported by the German Research Foundation (DFG) within the Joint Sino-German research project Control and Optimization for Eventtriggered Networked Autonomous Multi-agent Systems (COVEMAS)

P. Budde gen. Dohmann and S. Hirche are with the Chair of Informationoriented Control, Technische Universität München, Munich, Germany, e-mail: \{pablo.dohmann, hirche\}@tum.de wireless in case of mobile systems. Common robot control strategies require a sampling rate of $1 \mathrm{kHz}$ or higher, which poses high requirements on the communication channel, in particular in wireless communication [6]. This load on the communication network further increases with an increasing number of agents. Although typically a low number of manipulators are involved in a single cooperative manipulation task, additional agents might share the communication channel. It is well known that high network load leads to network congestion and, as a result, to packet loss and delays, which are detrimental to performance and can even destabilize the overall system. Additionally, in scenarios with battery-driven communication nodes, the lifetime is reduced by continuous signal transmission. It is important to develop approaches that can flexibly react to the communication constraints imposed by the application scenario. Event-triggered updating, where agents exchange data only when a certain triggering condition is met, is a solution to reduce the communication network load [7]. This has not been exploited yet in the context of cooperative manipulation. In general, the problem of distributed control for cooperative manipulation over communication networks is still a largely open problem.

In this article we will show that distributed control of multi-robot systems offers significant functional advantages for cooperative manipulation tasks since multiple different control objectives can be fulfilled simultaneously. We also address the issue of communication efficiency by introducing an event-triggered distributed control solution. Particular focus is on robust solutions, via passivity-based control, enabling manipulators to interact with possibly unknown objects and environments.

\section{A. Related Work}

In the following, a short overview of related works in the respective areas of cooperative manipulation and eventtriggered control is given.

a) Cooperative Manipulation: The goal in cooperative manipulation is typically two-fold; (i) the object should follow the desired trajectory and (ii) a suitable grasping force should be applied. Here suitable means that the force should be high enough such that the grasp is rigid and no slippage occurs while avoiding high interaction wrenches, which could damage the object. Common approaches to achieve those goals are classified as hybrid motion/force schemes and as impedancebased schemes. In hybrid motion/force control approaches the grasping forces are actively controlled in a space orthogonal to the desired motion [8]. The main problem arises in the 
computation of the actual grasping forces since they generally cannot be obtained without the knowledge of all the individual end-effector forces. Those can either be directly estimated [9] or are obtained by subtracting object dynamics, estimated in distributed fashion [10] from the measured forces. As a result, delays and errors due to the estimation of internal forces lead to a deteriorated performance in hybrid motion/force schemes. In contrast, with impedance-based schemes a forcemotion relationship is imposed on the system. Typically, those approaches come with guaranteed stability when in contact with a passive environment due to the passive nature of the impedance dynamics. Earlier works propose impedance control on object level [11] or based on internal forces applied to the object [12]. Both approaches can be combined to form a unified control framework for cooperative manipulation [13]. The dynamic behavior can be decoupled into a relative and cooperative part, which are directly related to the two sub-tasks of grasp maintenance and object tracking, and controlled separately by a central unit [14]. In contrast, with the decentralized control framework [15], [16], each manipulator is locally controlled via a local impedance-based scheme. As a result, the behaviors in the respective subspaces emerge as a consequence of the individual end-effector impedances and cannot be designed independently. However, object tracking and grasp maintenance may have different and conflicting control goals, for example, high precision for object tracking and high compliance for the grasp maintenance sub-task to compensate for uncertainties in the system. An impedance scheme, where the behaviors in the sub-spaces can be independently designed with a non-centralized control architecture, has not yet been introduced.

b) Event-Triggered Control: A general introduction to event-triggered and self-triggered control for single-agent systems can be found in [17]. In [18] a survey on recent advances for general nonlinear systems is presented. Various canonical problems in the area of multi-agent systems are solved in event-triggered fashion for simple agent dynamics (single/double integrator, linear) [19]-[21]. Nonlinear agent dynamics in multi-agent systems are considered in [22], [23] and an event-triggered control law, considering the nonlinear dynamics of flying robots, is proposed in [24]. As a special case of non-linear systems, Euler-Lagrange systems are recently considered in the context of event-triggered control [25]-[28]. In [28] an adaptive event-triggered control law for the leader-follower consensus of multiple EulerLagrange systems is presented and extended by additional model-independent algorithms. The so-called Zeno behavior, where an event is triggered infinitely often in a limited time interval, is a controversial topic on its own [29]. With typical triggering-conditions, the closer the agent gets to the origin the more events are triggered, which is unproblematic as long as it is the equilibrium point. However, this is not necessarily true since zero crossings can occur when an agent crosses the origin during its transient phase, leading to Zeno behavior. In order to avoid Zeno behavior during zero crossings, a decreasing state independent term is added to the triggering function. Previous approaches on event-triggered control in Euler-Lagrange systems do not consider external forces in the manipulator dynamics and are therefore not applicable to cooperative manipulation, where a certain interaction force is desired to maintain the grasp of the object. Passivity is often used to achieve robust stability for interaction tasks in EulerLagrange systems [30]. Passivity analysis of event-triggered systems is presented in [31]-[34] and based on the passivity indexes of plant and controller event-triggering conditions are proposed. However, those works only consider singleagent systems and are not applicable in distributed multi-agent systems.

While event-triggering in Lagrangian systems has recently gained attention, only general problems like consensus and leader-follower tasks have been analyzed. Event-triggering in cooperative manipulation is a completely unexplored topic. In addition, to the best knowledge of the authors event-triggering in multi-robot systems has never been experimentally validated, making the results presented in this article the first of its kind.

\section{B. Contributions}

The contribution of this work is twofold. First, a novel distributed control law for cooperative manipulation is proposed, allowing for more flexibility in the control design, i.e. differing control goals for object tracking and grasp maintenance. This is achieved by introducing communication between locally impedance-controlled end-effectors, which allows them to synchronize their errors. We characterize the performance of the system under the influence of disturbances in terms of $\mathcal{L}_{2}$ stability and provide upper bounds on the tracking error. An extensive discussion about how the communication between agents benefits the control design in a cooperative manipulation task is provided, and the influence of different communication topologies is discussed. The second contribution is a novel event-triggered update strategy to reduce the communication rate for the distributed control. We show that the triggering can be interpreted as an additional bounded and vanishing disturbance to the system. Applying passivity-based design techniques and Lyapunov theory the stability of the complete system can be shown even when in contact with an unknown object and environment. Finally, the results are illustrated in simulations for various communication topologies and validated in experiments, where a typical pick and place task with objects of varying stiffness is performed.

The remainder of this article is structured as follows. The system dynamics and a model of interaction between robot and object are presented in Section [II The distributed control law is developed first with time-continuous communication in Section IIII and extended by an event-triggered strategy in Section IV Finally, the approach is validated in simulations in Section $\mathrm{V}$ and an experiment in Section VI

\section{Notation}

Throughout this article any vector $\boldsymbol{a}_{i}$ or matrix $\boldsymbol{A}_{i}$ indexed with $i=[1, \ldots, N]$ refers to the $i$ th agent, while the absence of an index indicates the stacked vector of all agents $\boldsymbol{a}=\left[\begin{array}{lll}\boldsymbol{a}_{1}^{T} & \ldots & \boldsymbol{a}_{N}^{T}\end{array}\right]^{T}$ or the block diagonal matrix 
$\boldsymbol{A}=\operatorname{blockdiag}\left(\boldsymbol{A}_{1}, \ldots, \boldsymbol{A}_{N}\right)$. The smallest and largest eigenvalue of a matrix $\boldsymbol{A}$ is denoted with $\lambda_{\min }(\boldsymbol{A})$ and $\lambda_{\max }(\boldsymbol{A})$, respectively. The $n \times 1$ vector $\mathbf{1}_{n}$ denotes a column vector full of ones while $\boldsymbol{I}_{n}$ is the $n \times n$ identity matrix and $\mathbf{0}_{n \times m}$ is the $n \times m$ zero matrix. The indexes are omitted if the dimensions are clear from context. The Kronecker product operator is denoted as $\otimes$. The Euclidean norm of a vector and the matrix norm, induced by the Euclidean norm, are denoted by $\|(\cdot)\|_{2}$. Finally, the $\mathcal{L}_{2}$ norm of a function is denoted as $\|(\cdot)\|_{\mathcal{L}_{2}}$.

\section{Multi-Robot System Model}

In this work we consider $N$ manipulators cooperatively manipulating an object. Following the Euler-Lagrange formalism, the dynamics of the $i$ th manipulator in task space are given as

$$
\boldsymbol{\Lambda}_{i}\left(\boldsymbol{x}_{i}\right) \ddot{\boldsymbol{x}}_{i}+\boldsymbol{C}_{i}\left(\boldsymbol{x}_{i}, \dot{\boldsymbol{x}}_{i}\right) \dot{\boldsymbol{x}}_{i}+\boldsymbol{g}_{i}\left(\boldsymbol{x}_{i}\right)=\boldsymbol{h}_{i}^{c}+\boldsymbol{h}_{i},
$$

where $\boldsymbol{\Lambda}_{i}\left(\boldsymbol{x}_{i}\right) \in \mathbb{R}^{3 \times 3}$ and $\boldsymbol{C}_{i}\left(\boldsymbol{x}_{i}, \dot{\boldsymbol{x}}_{i}\right) \in \mathbb{R}^{3 \times 3}$ are the inertia matrix and Coriolis/centrifugal terms, respectively; $\boldsymbol{g}_{i}\left(\boldsymbol{x}_{i}\right) \in$ $\mathbb{R}^{3}$ are the gravitational forces, $\boldsymbol{h}_{i}^{c} \in \mathbb{R}^{3}$ are the control inputs, and $\boldsymbol{x}_{i}, \dot{\boldsymbol{x}}_{i}, \ddot{\boldsymbol{x}}_{i} \in \mathbb{R}^{3}$ are the position, velocity and acceleration of the $i$ th end-effector. The total external applied force $\boldsymbol{h}_{i} \in \mathbb{R}^{3}$ is measured at the tip of the $i$ th end-effector and includes the contact with the object and the environment. When interacting with an unknown environment, the well known impedance-control approach provides a safe framework for robotic manipulation [35]. By defining the tracking error as

$$
\boldsymbol{z}_{i}=\boldsymbol{x}_{i}-\boldsymbol{x}_{i}^{d}
$$

with the desired trajectory $\boldsymbol{x}_{i}^{d}$ for the $i$ th manipulator, the desired error dynamics are given as

$$
\boldsymbol{M}_{i} \ddot{z}_{i}+\boldsymbol{D}_{i} \dot{z}_{i}+\boldsymbol{K}_{i} \boldsymbol{z}_{i}=\boldsymbol{h}_{i}+\boldsymbol{h}_{i}^{s}+\tilde{\boldsymbol{h}}_{i}
$$

with symmetric positive definite desired inertia $\boldsymbol{M}_{i} \in \mathbb{R}^{3 \times 3}$, damping $\boldsymbol{D}_{i} \in \mathbb{R}^{3 \times 3}$ and stiffness $\boldsymbol{K}_{i} \in \mathbb{R}^{3 \times 3}$. The term $\boldsymbol{h}_{i}^{s} \in$ $\mathbb{R}^{3}$ is an additive force, which can be used as an additional control input, which is exploited later and $\tilde{\boldsymbol{h}}_{i} \in \mathbb{R}^{3}$ is a disturbance force. Stacking the individual dynamics (3), the system consisting of all $N$ manipulators can be compactly written as

$$
M \ddot{z}+D \dot{z}+K z=h+h^{s}+\tilde{h} .
$$

Common approaches to achieve the desired dynamics (4) can be distinguished into force- and position-based impedance schemes. In force-based approaches the desired actuator torques are obtained by feeding back position and velocity of the end-effector and are transformed to the joint-space with known Jacobian. An accurate model of the manipulator dynamics is required in order to compensate for the nonlinear terms $\boldsymbol{C}_{i}\left(\boldsymbol{x}_{i}, \dot{\boldsymbol{x}}_{i}\right) \dot{\boldsymbol{x}}_{i}$ and $\boldsymbol{g}_{i}\left(\boldsymbol{x}_{i}\right)$ in (1). In addition, force-feedback can be incorporated in order to shape the desired inertia of the end-effector. In contrast, in positionbased impedance approaches, measured forces are fed back and the desired motion is obtained according to (4). A position controller is then used to track the desired motion. For more information on the individual approaches, the interested reader is referred to [36], [37]. Under the assumption of perfect feedback linearization for the force-based and perfect tracking of the virtual end-effector trajectory for the position-based impedance approaches, the closed-loop dynamics of the multirobot system can be expressed by (4), making the results presented in this article independent of the actual implementation. Depending on the implementation the disturbance forces $\tilde{h}$ can thus include uncertainty in the manipulator dynamics for the feedback linearization, as well as measurement errors in the forces $\boldsymbol{h}$ and/or tracking errors of the position controller. While we allow for unknown and possibly flexible objects as well as contact with the environment, we pose the following assumptions on the grasp and the externally applied forces $\boldsymbol{h}$.

\section{Assumption 1:}

1) The desired trajectory $\boldsymbol{x}^{d}$ is chosen such that the grasp can be assumed as rigid and no slippage occurs.

2) The external forces $\boldsymbol{h}$ applied to the system are generated by a passive map with input $\dot{\boldsymbol{x}}$ and output $-\boldsymbol{h}$, i.e. there exists a positive semi-definite function $V_{e}$ such that $\dot{V}_{e} \leq-\dot{\boldsymbol{x}}^{T} \boldsymbol{h}$. Additionally, the force $\boldsymbol{h}$ resulting from a bounded velocity $\dot{x}$ is bounded.

With the above assumption the combined system of object and environment is assumed to be passive. This is the case if both the environment and object are passive. While passivity of the environment is a typical assumption in robotic applications, also the passivity of the object is not restrictive since physical objects are modeled via Euler-Lagrange equations at the contact points [38], which naturally form passive systems [39]. This, however, only holds if the contact points do not change during manipulation, which is provided by the rigid grasp assumption. A strategy for choosing $\boldsymbol{x}^{d}$ such that the first item of Assumption 1 is fulfilled is provided in Section III-C

\section{A. Dynamics Decoupling}

Recall the two tasks for cooperative manipulation discussed in Section [1. namely i) object tracking and ii) grasp maintenance. In order to analyze the achievement of the two subtasks, similar to [40] the system dynamics are decoupled into a cooperative sub-system, related to task i), and a relative subsystem, related to task ii). The cooperative state is defined as the geometric center of the grasping points as

$$
\boldsymbol{z}_{c}=\frac{1}{N} \sum_{i=1}^{N} \boldsymbol{z}_{i}=\boldsymbol{G} \boldsymbol{z},
$$

where $G \in \mathbb{R}^{3 \times 3 N}$ is defined as

$$
\boldsymbol{G}=\frac{1}{N} \mathbf{1}_{N}^{T} \otimes \boldsymbol{I}_{3} .
$$

The states evolving in the null-space of $\boldsymbol{G}$ are the relative states given as

$$
\boldsymbol{z}_{r}=\mathbf{N} \boldsymbol{z}
$$

with a full row-rank null-space projector $\boldsymbol{N}=\boldsymbol{F} \otimes \boldsymbol{I}_{3}$, where $\boldsymbol{F} \in \mathbb{R}^{(N-1) \times N}$ is chosen such that $\boldsymbol{F} \mathbf{1}_{N}=\mathbf{0}_{N-1}$ and thus $N \boldsymbol{G}^{T}=\mathbf{0}_{3(N-1) \times 3 N}$. We can now write

$$
\boldsymbol{z}=\left[\begin{array}{ll}
\boldsymbol{G}^{\dagger} & \boldsymbol{N}^{\dagger}
\end{array}\right]\left[\begin{array}{l}
\boldsymbol{z}_{c} \\
\boldsymbol{z}_{r}
\end{array}\right]
$$


where $G^{\dagger}$ and $N^{\dagger}$ denote the Moore-Penrose inverse of $G$ and $N$, respectively. Equivalent transformations can be made for the original system and desired states $\boldsymbol{x}, \boldsymbol{x}^{d}$ as

$$
\left[\begin{array}{l}
\boldsymbol{x}_{c} \\
\boldsymbol{x}_{r}
\end{array}\right]=\left[\begin{array}{c}
\boldsymbol{G} \\
\boldsymbol{N}
\end{array}\right] \boldsymbol{x}, \quad\left[\begin{array}{c}
\boldsymbol{x}_{c}^{d} \\
\boldsymbol{x}_{r}^{d}
\end{array}\right]=\left[\begin{array}{c}
\boldsymbol{G} \\
\boldsymbol{N}
\end{array}\right] \boldsymbol{x}^{d}
$$

Remark 1: For the sake of exposition we assume homogeneous impedance parameters. It should be noted that this is solely required for the intuitive representation in terms of cooperative and relative behaviors. In case of nonhomogeneous parameters, the decomposition is still possible with energetically-conservative coupled behaviors [41]. Our approach, including stability results, straightforwardly extends to the case of non-homogeneous impedances.

Due to the homogeneity every impedance parameter $\boldsymbol{A} \in\{\boldsymbol{M}, \boldsymbol{D}, \boldsymbol{K}\}$ is simplified as

$$
\boldsymbol{A}=\boldsymbol{I}_{N} \otimes \boldsymbol{A}_{i} .
$$

Due to the properties of the Kronecker product

$$
\boldsymbol{N}^{\dagger}=\boldsymbol{F}^{\dagger} \otimes \boldsymbol{I}_{3}, \quad \boldsymbol{G}^{\dagger}=\mathbf{1}_{N} \otimes \boldsymbol{I}_{3}
$$

holds and it can be shown that

$$
\left(\boldsymbol{N}^{\dagger}\right)^{T} \boldsymbol{A} \boldsymbol{G}^{\dagger}=\mathbf{0}_{3(N-1) \times 3}, \quad\left(\boldsymbol{G}^{\dagger}\right)^{T} \boldsymbol{A} \boldsymbol{N}^{\dagger}=\mathbf{0}_{3 \times 3(N-1)} .
$$

By substituting [8) in (4), left-multiplying by $\left[\begin{array}{c}\left(\boldsymbol{G}^{\dagger}\right)^{T} \\ \left(\boldsymbol{N}^{\dagger}\right)^{T}\end{array}\right]$, and by using the property (12), the decoupled dynamics in the respective sub-spaces are obtained as

$$
\begin{aligned}
\boldsymbol{M}_{c} \ddot{\boldsymbol{z}}_{c}+\boldsymbol{D}_{c} \dot{\boldsymbol{z}}_{c}+\boldsymbol{K}_{c} \boldsymbol{z}_{c}=\boldsymbol{h}_{c}+\tilde{\boldsymbol{h}}_{c}+\boldsymbol{h}_{c}^{s} \\
\boldsymbol{M}_{r} \ddot{z}_{r}+\boldsymbol{D}_{r} \dot{\boldsymbol{z}}_{r}+\boldsymbol{K}_{r} \boldsymbol{z}_{r}=\boldsymbol{h}_{r}+\tilde{\boldsymbol{h}}_{r}+\boldsymbol{h}_{r}^{s},
\end{aligned}
$$

where a matrix $\boldsymbol{A} \in\{\boldsymbol{M}, \boldsymbol{D}, \boldsymbol{K}\}$ and a force $\boldsymbol{a} \in\left\{\boldsymbol{h}, \tilde{\boldsymbol{h}}, \boldsymbol{h}^{s}\right\}$ indexed by $c, r$ are defined as

$$
\begin{array}{ll}
\boldsymbol{A}_{c}=\left(\boldsymbol{G}^{\dagger}\right)^{T} \boldsymbol{A} \boldsymbol{G}^{\dagger}, & \boldsymbol{a}_{c}=\left(\boldsymbol{G}^{\dagger}\right)^{T} \boldsymbol{a}, \\
\boldsymbol{A}_{r}=\left(\boldsymbol{N}^{\dagger}\right)^{T} \boldsymbol{A} \boldsymbol{N}^{\dagger}, & \boldsymbol{a}_{r}=\left(\boldsymbol{N}^{\dagger}\right)^{T} \boldsymbol{a} .
\end{array}
$$

\section{Distributed CoOperative Manipulation}

In the following we will exploit communication between the individual manipulators to obtain more flexibility in the design of the control laws for the two sub-spaces. This is done by allowing the agents to communicate the individual tracking errors $\dot{\boldsymbol{z}}_{i}, \boldsymbol{z}_{i}$ and synchronize them with their neighbors via the additional control input $\boldsymbol{h}^{s}$ in (4).

\section{A. Communication Graph Model}

The communication structure of $N$ interacting agents is modeled by a weighted undirected graph $\mathcal{G}=(\mathcal{V}, \mathcal{E})$, with vertex set $\mathcal{V}=\{1, \ldots, N\}$ representing the $N$ agents and edge set $\mathcal{E} \subseteq \mathcal{V} \times \mathcal{V}$, representing the connection between agents. The neighborhood of agent $i$ is defined as $N_{i}=\{j \in$ $\mathcal{V} \mid(i, j) \in \mathcal{E}\}$. In this work we consider an undirected graph, i.e. if $(i, j) \in \mathcal{E} \Rightarrow(j, i) \in \mathcal{E}$. In contrast to typical consensus and formation problems, we do not assume a connected communication graph, i.e. there does not necessarily exist a path between any two vertices $i$ and $j$. The $N \times|\mathcal{E}|$ incidence matrix $\boldsymbol{H}=\left\{h_{i k}\right\}$ of the graph $\mathcal{G}$ is given as

$$
h_{i k}= \begin{cases}1 & \text { if edge } k \text { enters node } i \\ -1 & \text { if } k \text { leaves node } i \\ 0 & \text { otherwise. }\end{cases}
$$

In this work we make use of a matrix weighted graph, extended to the three spacial dimensions. Then the $3 N \times 3 N$ Laplacian matrix is obtained as

$$
\boldsymbol{L}_{A}=\left(\boldsymbol{H} \otimes \boldsymbol{I}_{3}\right)^{T} \boldsymbol{A}\left(\boldsymbol{H} \otimes \boldsymbol{I}_{3}\right),
$$

where the weight matrix is defined as $\boldsymbol{A}=\operatorname{blockdiag}\left(\boldsymbol{A}_{k}\right)$ with individual weight $\boldsymbol{A}_{k} \in \mathbb{R}^{3 \times 3}$ for each edge $k \in \mathcal{E}$. Note that we do not require positive definite weights and thus the Laplacian matrix itself is not necessary positive definite as typically assumed in consensus and formation control problems.

\section{B. Error Synchronization}

In this work agents exchange their position and velocity errors via the additional control input $\boldsymbol{h}_{i}^{s}$, defined as

$$
\boldsymbol{h}_{i}^{s}=\sum_{j \in N_{i}} \boldsymbol{D}_{i j}^{s}\left(\dot{\boldsymbol{z}}_{j}-\dot{\boldsymbol{z}}_{i}\right)+\sum_{j \in N_{i}} \boldsymbol{K}_{i j}^{s}\left(\boldsymbol{z}_{j}-\boldsymbol{z}_{i}\right),
$$

where $\boldsymbol{D}_{i j}^{s} \in \mathbb{R}^{3 \times 3}, \boldsymbol{K}_{i j}^{s} \in \mathbb{R}^{3 \times 3}$ are design parameters fulfilling following assumption.

Assumption 2: The parameters in (18) are chosen such that $\boldsymbol{D}_{i j}^{s}=\boldsymbol{D}_{j i}^{s}$ and $\boldsymbol{K}_{i j}^{s}=\boldsymbol{K}_{j i}^{s}$ and the individual matrices $\boldsymbol{D}_{i j}^{s}, \boldsymbol{K}_{i j}^{s}$ are symmetric.

Remark 2: In the following it will be shown that the synchronization controller can be interpreted as additional stiffness and damping between neighboring end-effectors. The symmetry assumption on the weight matrices $\boldsymbol{D}_{i j}^{s}, \boldsymbol{K}_{i j}^{s}$ is a typical requirement on the parameters of impedance-based control schemes, describing a notion of physical feasibility. Note that besides the symmetry assumption, no further restrictions are posed on the parameters. This implies that spatially (de-)coupled parameters and different weights for different edges are possible.

Based on Assumption 2 we can exchange the indexes $i j$ and $j i$ by the index $k \in \mathcal{E}$, which denotes the edge connecting agents $i$ and $j$. Defining $\boldsymbol{L}_{D} \in \mathbb{R}^{3 N \times 3 N}$ and $\boldsymbol{L}_{K} \in \mathbb{R}^{3 N \times 3 N}$ as in (17) with the $3|\mathcal{E}| \times 3|\mathcal{E}|$ weight matrices $\boldsymbol{D}^{s}=\operatorname{blockdiag}\left(\boldsymbol{D}_{1}^{s}, \cdots, \boldsymbol{D}_{|\mathcal{E}|}^{s}\right)$ and $\boldsymbol{K}^{s}=$ blockdiag $\left(\boldsymbol{K}_{1}^{s}, \cdots, \boldsymbol{K}_{|\mathcal{E}|}^{s}\right)$ replacing $\boldsymbol{A}$ in (17), the synchronization input in (4) is compactly written as

$$
\boldsymbol{h}^{s}=-\boldsymbol{L}_{D} \dot{\boldsymbol{z}}-\boldsymbol{L}_{K} \boldsymbol{z},
$$

resulting in the coupled closed-loop dynamics

$$
M \ddot{z}+D_{a} \dot{z}+K_{a} z=h+\tilde{h},
$$

where $\boldsymbol{D}_{a}=\boldsymbol{D}+\boldsymbol{L}_{D}$ and $\boldsymbol{K}_{a}=\boldsymbol{K}+\boldsymbol{L}_{K}$. Projecting 19 into the corresponding sub-spaces, one obtains

$$
\begin{aligned}
& \boldsymbol{h}_{c}^{s}=-\left(\boldsymbol{G}^{\dagger}\right)^{T} \boldsymbol{L}_{D} \dot{\boldsymbol{z}}-\left(\boldsymbol{G}^{\dagger}\right)^{T} \boldsymbol{L}_{K} \boldsymbol{z} \\
& \boldsymbol{h}_{r}^{s}=-\left(\boldsymbol{N}^{\dagger}\right)^{T} \boldsymbol{L}_{D} \dot{\boldsymbol{z}}-\left(\boldsymbol{N}^{\dagger}\right)^{T} \boldsymbol{L}_{K} \boldsymbol{z} .
\end{aligned}
$$


It should be noted that

$$
\operatorname{span}\left\{\mathbf{1}_{N} \otimes \boldsymbol{I}_{3}\right\} \subseteq \operatorname{null}\left(\boldsymbol{L}_{A}\right)
$$

and since $\boldsymbol{G}^{\dagger}=\mathbf{1}_{N} \otimes \boldsymbol{I}_{3}$, we can conclude that $\left(\boldsymbol{G}^{\dagger}\right)^{T} \boldsymbol{L}_{l}=$ $\mathbf{0}_{3 N}, \forall l \in\{D, K\}$ and $\boldsymbol{h}_{c}^{s}=\mathbf{0}_{3 N}$ follows immediately. Substituting (8) and its derivative into (22) results in

$$
\boldsymbol{h}_{r}^{s}=-\left(\boldsymbol{N}^{\dagger}\right)^{T} \boldsymbol{L}_{D} \boldsymbol{N}^{\dagger} \dot{\boldsymbol{z}}_{r}-\left(\boldsymbol{N}^{\dagger}\right)^{T} \boldsymbol{L}_{K} \boldsymbol{N}^{\dagger} \boldsymbol{z}_{r}
$$

since again the cooperative term vanishes. Combined with 13 and (14), the cooperative and relative impedance behaviors, including the synchronization control, are obtained as

$$
\begin{aligned}
& \boldsymbol{M}_{c} \ddot{\boldsymbol{z}}_{c}+\boldsymbol{D}_{c} \dot{\boldsymbol{z}}_{c}+\boldsymbol{K}_{c} \boldsymbol{z}_{c}=\boldsymbol{h}_{c}+\tilde{\boldsymbol{h}}_{c} \\
& \boldsymbol{M}_{r} \ddot{\boldsymbol{z}}_{r}+\boldsymbol{D}_{e} \dot{\boldsymbol{z}}_{r}+\boldsymbol{K}_{e} \boldsymbol{z}_{r}=\boldsymbol{h}_{r}+\tilde{\boldsymbol{h}}_{r},
\end{aligned}
$$

with the effective relative stiffness and damping

$$
\begin{aligned}
& \boldsymbol{K}_{e}=\left(\boldsymbol{N}^{\dagger}\right)^{T}\left(\boldsymbol{K}+\boldsymbol{L}_{K}\right) \boldsymbol{N}^{\dagger} \\
& \boldsymbol{D}_{e}=\left(\boldsymbol{N}^{\dagger}\right)^{T}\left(\boldsymbol{D}+\boldsymbol{L}_{D}\right) \boldsymbol{N}^{\dagger} .
\end{aligned}
$$

As a result, the cooperative behavior (i.e. object tracking) is not affected by the synchronization input, while the resulting relative behavior (i.e. grasp maintenance) is an impedance behavior with modified relative stiffness and damping. Consequently, by tuning the respective weight matrices $\boldsymbol{K}_{k}^{s}$ and $\boldsymbol{D}_{k}^{s}, \forall k \in \mathcal{E}$ the relative parameters can be set without changing the cooperative parameters. However, this modification of the relative parameters is only possible along the edges of the underlying communication graph, characterized by the $N \times N$ reduced graph Laplacian matrix

$$
\boldsymbol{L}^{*}=\boldsymbol{H}^{T} \boldsymbol{H}
$$

with the incidence matrix $H$ as defined in (16). In the following we will investigate the passivity and stability properties of the overall system. The following lemma and definition will be helpful for that.

Lemma 1: If

$$
\left.\begin{array}{l}
\lambda_{\min }\left(\boldsymbol{K}_{k}^{s}\right)>-\frac{\lambda_{\min }(\boldsymbol{K})}{\lambda_{\max }\left(\boldsymbol{L}^{*}\right)} \\
\lambda_{\min }\left(\boldsymbol{D}_{k}^{s}\right)>-\frac{\lambda_{\min }(\boldsymbol{D})}{\lambda_{\max }\left(\boldsymbol{L}^{*}\right)}
\end{array}\right\}, \forall k \in \mathcal{E}
$$

then the matrices $\boldsymbol{D}_{e}, \boldsymbol{K}_{e}$ as defined in 27) are positive definite.

Proof: From (27) it can be seen that if $\boldsymbol{K}^{s}$ is positive semidefinite, $\boldsymbol{K}_{e}$ is positive definite. In the case that $\lambda_{\min }\left(\boldsymbol{K}^{s}\right)<0$, we can write $\lambda_{\min }\left(\boldsymbol{L}_{K}\right) \geq$ $\lambda_{\max }\left(\boldsymbol{L}^{*}\right) \lambda_{\min }\left(\boldsymbol{K}^{s}\right)$. Recall that $\boldsymbol{K}^{s}$ is a block diagonal matrix with $\boldsymbol{K}_{k}^{s}$ on the diagonals, where $k$ denotes the edges of the graph, thus $\lambda_{\min }\left(\boldsymbol{K}^{s}\right)=\min _{k \in \mathcal{E}} \lambda_{\min }\left(\boldsymbol{K}_{k}^{s}\right)$. In order to ensure positive definiteness of $\boldsymbol{K}_{e}{ }^{k}$,

$$
\lambda_{\min }(\boldsymbol{K})+\lambda_{\max }\left(\boldsymbol{L}^{*}\right) \lambda_{\min }\left(\boldsymbol{K}_{k}^{s}\right)>0
$$

must hold for all edges $k$, which is achieved by (29). Following the same argumentation positive definiteness of $\boldsymbol{D}_{e}$ can be shown.

Definition 1 ([42]]): A dynamical system with state $\boldsymbol{x} \in \mathbb{R}^{n}$, input $\boldsymbol{u} \in \mathbb{R}^{p}$, and output $\boldsymbol{y} \in \mathbb{R}^{p}$ is said to be output strictly passive (OSP) with passivity index $\rho$ if there exists a continuously differentiable, positive semidefinite function $V(\boldsymbol{x})$ such that

$$
\dot{V} \leq \boldsymbol{u}^{T} \boldsymbol{y}-\rho \boldsymbol{y}^{T} \boldsymbol{y}
$$

and $\rho>0$.

Now we are in the position to make a statement on the passivity and stability properties of the overall system.

Theorem 1: Consider multiple impedance-controlled manipulators with error dynamics (4) cooperatively manipulating an object with the control input $h^{s}$ as given in 19 and under Assumption 2 If the parameters are chosen according to 29 ) then the coupled manipulator dynamics 20 ) are outputstrictly-passive with input-output pair $[\boldsymbol{u}, \boldsymbol{y}]=[\boldsymbol{h}+\tilde{\boldsymbol{h}}, \dot{\boldsymbol{z}}]$ and passivity index $\rho=\lambda_{\min }\left(\boldsymbol{D}+\boldsymbol{L}_{D}\right)$.

Proof: Consider the Lyapunov candidate

$$
V_{c}=\frac{1}{2}\left[\begin{array}{c}
\boldsymbol{z} \\
\dot{\boldsymbol{z}}
\end{array}\right]^{T} \underbrace{\left[\begin{array}{cc}
\boldsymbol{K}_{a}+c \boldsymbol{D}_{a} & c \boldsymbol{M} \\
c \boldsymbol{M} & \boldsymbol{M}
\end{array}\right]}_{W}\left[\begin{array}{c}
\boldsymbol{z} \\
\dot{\boldsymbol{z}}
\end{array}\right] .
$$

It can be shown using the Schur complement that $V_{c}$ is positive definite if $\frac{\lambda_{d}-\sqrt{\lambda_{d}^{2}+4 \lambda_{k} \lambda_{m}}}{2 \lambda_{m}}<c<\frac{\lambda_{d}+\sqrt{\lambda_{d}^{2}+4 \lambda_{k} \lambda_{m}}}{2 \lambda_{m}}$, where $\lambda_{k}=\lambda_{\min }\left(\boldsymbol{K}_{a}\right), \lambda_{d}^{2 \lambda_{m}}=\lambda_{\min }\left(\boldsymbol{D}_{a}\right)$ and $\lambda_{m}^{2 \lambda_{m}}=\lambda_{\max }(\boldsymbol{M})$. Taking the derivative of $V_{c}$ along the solutions of [20], one obtains

$$
\dot{V}_{c}=\boldsymbol{u}^{T}(\dot{\boldsymbol{z}}+c \boldsymbol{z})-\dot{\boldsymbol{z}}^{T}\left(\boldsymbol{D}_{a}-c \boldsymbol{M}\right) \dot{\boldsymbol{z}}-c \boldsymbol{z}^{T} \boldsymbol{K}_{a} \boldsymbol{z} .
$$

Now define $V_{0}$ as in (32) with $c=0$, resulting in

$$
\dot{V}_{0} \leq \boldsymbol{u}^{T} \dot{\boldsymbol{z}}-\lambda_{d}\|\dot{\boldsymbol{z}}\|_{2}^{2},
$$

from which the OSP property can be immediately concluded.

Remark 3: Note that due to the OSP property, if $\tilde{\boldsymbol{h}}=\mathbf{0}_{3 N}$, the origin is stable according to [42, Lemma 6.6]. However, asymptotic stability of the origin can generally not be shown since, due to the unknown dynamics of the object and environment, the system is not necessarily zero-state observable according to [42, Definition 6.5]. However, due to the interconnection properties of passive systems, it is still possible to show that $\ddot{z}_{l}, \dot{z}_{l} \rightarrow \mathbf{0}$ and thus $\boldsymbol{z}_{l}$ converges to a constant value $\boldsymbol{z}_{l}^{*}$, depending on the object grasped and the environmental influence via the interaction force $h$ as

$$
\left[\begin{array}{l}
\boldsymbol{z}_{c}^{*} \\
\boldsymbol{z}_{r}^{*}
\end{array}\right]=\left[\begin{array}{l}
\boldsymbol{K}_{c}^{-1} \boldsymbol{h}_{c} \\
\boldsymbol{K}_{e}^{-1} \boldsymbol{h}_{r}
\end{array}\right] .
$$

Based on the passivity properties we can provide following performance bounds in terms of the resulting $\mathcal{L}_{2}$ gain.

Corollary 1: Let Assumption 1 and the conditions of Theorem 1 hold. Then the coupled manipulator dynamics (20) are $\mathcal{L}_{2}$ stable with

$$
\begin{aligned}
& \|\dot{\boldsymbol{z}}\|_{\mathcal{L}_{2}} \leq \gamma_{1}\|\tilde{\boldsymbol{h}}\|_{\mathcal{L}_{2}}+\alpha_{\dot{\boldsymbol{z}}} \\
& \|\boldsymbol{z}\|_{\mathcal{L}_{2}} \leq \gamma_{2}\|\boldsymbol{h}\|_{\mathcal{L}_{2}}+\gamma_{3}\|\tilde{\boldsymbol{h}}\|_{\mathcal{L}_{2}}+\alpha_{\boldsymbol{z}},
\end{aligned}
$$

where $\alpha_{\dot{z}}, \alpha_{\boldsymbol{z}}$ are positive scalars and

$$
\gamma_{1}=\frac{1}{\lambda_{d}}, \quad \gamma_{2}=\frac{1}{\lambda_{k}}, \quad \gamma_{3}=\frac{1}{\lambda_{k}}+\frac{\sqrt{\left(1+\lambda_{d}\right) \lambda_{m}}}{\lambda_{d} \sqrt{\lambda_{k}}} .
$$


Proof: The first bound (36) follows directly from the OSP property [42, Lemma 6.5]. Regarding (37) define $V_{1}$ as in (32) with $c=\frac{\lambda_{d}}{\lambda_{m}}$. The derivative of $V_{1}+V_{e}$ can be obtained as

$$
\begin{aligned}
& \dot{V}_{1}+\dot{V}_{e} \leq \frac{\lambda_{d}}{\lambda_{m}} \boldsymbol{u}^{T} \boldsymbol{z}+\tilde{\boldsymbol{h}}^{T} \dot{\boldsymbol{z}}-\frac{\lambda_{d} \lambda_{k}}{\lambda_{m}}\|\boldsymbol{z}\|_{2}^{2} \\
& \leq \frac{1}{2 \lambda_{d}}\|\tilde{\boldsymbol{h}}\|_{2}^{2}+\frac{\lambda_{d}}{2}\|\dot{\boldsymbol{z}}\|_{2}^{2}-\frac{\lambda_{d} \lambda_{k}}{2 \lambda_{m}}\|\boldsymbol{z}\|_{2}^{2}+\frac{\lambda_{d}}{2 \lambda_{m} \lambda_{k}}\|\boldsymbol{u}\|_{2}^{2}
\end{aligned}
$$

where the second line follows from Young's inequality. Integrating above inequality and solving for $\|\boldsymbol{z}\|_{\mathcal{L}_{2}}$ and substituting (36), results in

$$
\begin{aligned}
\|\boldsymbol{z}\|_{\mathcal{L}_{2}} & \leq\left(\frac{\sqrt{\left(1+\lambda_{d}\right) \lambda_{m}}}{\lambda_{d} \sqrt{\lambda_{k}}}+\frac{1}{\lambda_{k}}\right)\|\tilde{\boldsymbol{h}}\|_{\mathcal{L}_{2}}+\frac{1}{\lambda_{k}}\|\boldsymbol{h}\|_{\mathcal{L}_{2}} \\
& +\sqrt{2 \frac{\lambda_{m}}{\lambda_{d} \lambda_{k}}\left(V_{0}(0)+V_{1}(0)+V_{e}(0)\right)}
\end{aligned}
$$

Remark 4: As shown in [41] the passivity properties and with that the performance bounds directly transfer to the cooperative and relative sub-spaces, considering the modified parameters.

By inspecting 29] the strength of the proposed control law becomes evident; the matrices $\boldsymbol{K}_{k}^{s}$ and $\boldsymbol{D}_{k}^{s}$ do not need to be positive definite in order to tune the effective relative parameters $\boldsymbol{K}_{e}$ and $\boldsymbol{D}_{e}$ in (27). The restriction (29) is solely to guarantee positive definiteness of the resulting effective parameters. In addition, Corollary 1 highlights the importance of the individual parameters when it comes to disturbance rejection, which will be discussed in Section III-D

Remark 5: For the sake of exposition only translational motion of the agents is considered in this article. The focus is on the additional flexibility to achieve the two control goals of object transfer and grasp maintenance independently by adding the synchronization control. The extension to full pose control, including orientations of the end-effector, can be achieved by employing a passivity-based pose synchronization framework like e.g. [43].

\section{Trajectory Generation}

The desired trajectory $\boldsymbol{x}^{d}$ can be obtained by individually designing a trajectory for the cooperative and relative behavior, considering the individual task requirements, and inverting (9). For the cooperative motion the desired trajectory $\boldsymbol{x}_{c}^{d}$ is simply given by the desired motion of the object center. For the relative behavior the set-point $\boldsymbol{x}_{r}^{d}$ can be chosen to achieve a desired grasping force. This, however, is only possible with precise knowledge of the object model, as shown in the following example.

Example 1: Generally, the total force $\boldsymbol{h}$ at the end-effectors can be written as summation of forces due to interaction with the object $\boldsymbol{h}^{o}$ and forces due to other external influences $\boldsymbol{h}^{e}$. Assume the object can be approximated by springs with stiffness $\boldsymbol{K}_{o}$ between the grasping points such that the resulting force at the $i$ th end-effector is obtained as the sum of the individual springs as

$$
\boldsymbol{h}_{i}^{o}=\boldsymbol{K}_{o} \sum_{j=1}^{N}\left(\boldsymbol{x}_{j}-\boldsymbol{x}_{i}+\boldsymbol{r}_{i j}\right),
$$

where $\boldsymbol{r}_{i j}=\boldsymbol{r}_{i}-\boldsymbol{r}_{j}=$ const is the displacement between the $i$ th and $j$ th grasping point $\boldsymbol{r}_{i}$ and $\boldsymbol{r}_{j}$ in the undeformed state. In compact form we can write

$$
\boldsymbol{h}^{o}=\boldsymbol{K}_{L}(\boldsymbol{r}-\boldsymbol{x}),
$$

where the object stiffness is given as

$$
\boldsymbol{K}_{L}=\left[\begin{array}{cccc}
(N-1) & -1 & \ldots & -1 \\
-1 & (N-1) & \cdots & -1 \\
\vdots & \ddots & & \vdots \\
\cdots & -1 & -1 & (N-1)
\end{array}\right] \otimes \boldsymbol{K}_{o}
$$

Note that the structure of $\boldsymbol{K}_{L}$ in (45) is that of a Laplacian matrix with the same null-space properties, and as a result, only the relative behavior of the system is affected. Assume that the object is in free motion and no disturbance is present, i.e. $\boldsymbol{h}^{e}=\widetilde{\boldsymbol{h}}=\mathbf{0}$. It can be shown that in this case, the system converges to the equilibrium point

$$
\left[\begin{array}{c}
\boldsymbol{x}_{c}^{*} \\
\boldsymbol{x}_{r}^{*}
\end{array}\right]=\left[\begin{array}{c}
\boldsymbol{x}_{c}^{d} \\
\left(\boldsymbol{K}_{e}+\boldsymbol{K}_{L, r}\right)^{-1}\left(\boldsymbol{K}_{e} \boldsymbol{x}_{r}^{d}+\boldsymbol{K}_{L, r} \boldsymbol{N} \boldsymbol{r}\right)
\end{array}\right],
$$

leading to the grasping force

$$
\boldsymbol{h}_{r}^{o}=\boldsymbol{K}_{L, r}\left(\boldsymbol{K}_{e}+\boldsymbol{K}_{L, r}\right)^{-1} \boldsymbol{K}_{e}\left(\boldsymbol{N} \boldsymbol{r}-\boldsymbol{x}_{r}^{d}\right)
$$

at steady state, where $\boldsymbol{K}_{L, r}$ is the object stiffness, transformed into the relative sub-space as (15). We can now solve (47) for $\boldsymbol{x}_{r}^{d}$ in order to obtain the set-point to achieve a desired grasp force $\boldsymbol{h}_{r}^{d}$ as

$$
\boldsymbol{x}_{r}^{d}=\boldsymbol{N} \boldsymbol{r}-\boldsymbol{K}_{e}^{-1}\left(\boldsymbol{K}_{e}+\boldsymbol{K}_{L, r}\right) \boldsymbol{K}_{L, r}^{-1} \boldsymbol{h}_{r}^{d} .
$$

The major drawback of choosing $\boldsymbol{x}_{r}^{d}$ in such a way is that precise knowledge of the object is required; in the above example this would be the object stiffness $\boldsymbol{K}_{o}$ as well as the geometric parameter $\boldsymbol{r}$. As a result, modeling errors can lead to deteriorated grasping forces $\boldsymbol{h}_{r}$, which are amplified by the relative stiffness $\boldsymbol{K}_{e}$. Alternatively, a constant desired force can be achieved by setting the relative stiffness $\boldsymbol{K}_{e}=\mathbf{0}$ and simply applying the desired force as feed-forward term. Assuming no other influences, i.e. $\boldsymbol{h}^{e}=\tilde{\boldsymbol{h}}=\mathbf{0}$, it can be easily verified that the desired force is tracked. However, since the relative behavior is not directly controlled as in centralized approaches, we can merely influence $\boldsymbol{K}_{e}$ via the synchronization term $\boldsymbol{h}^{s}$. In conclusion, generally low relative stiffness is desired in order to increase performance in the grasping task.

Remark 6: With the proposed strategy the desired trajectory is obtained by the individual design in the respective sub-spaces and inverting the second equation of 99. As a result, knowledge of the desired trajectory is given as central information. In this work, we assume that the task is known beforehand and that the trajectory can be generated prior to task execution and locally stored with each agent. Whenever 
this is not possible, e.g. in a teleoperation scenario, distributed estimation algorithms can be used to recover the information at every agent $[3]$.

\section{Guidelines for Controller Design}

This section provides guidelines on how to chose the individual parameters in order to exploit the presented results. Based on (46) and an exact environment model, it may be possible to compute a set-point $\boldsymbol{x}^{d}$ to achieve perfect force tracking. Alternatively, in the case of an unknown environment, low stiffness is desired in order to achieve good force tracking. By recalling the two goals for cooperative manipulation, force tracking is often of higher priority for the relative task, while for the cooperative task accurate motion tracking is often of higher priority. In this scenario a low relative stiffness $\boldsymbol{K}_{e}$ and a high cooperative stiffness $\boldsymbol{K}_{c}$ are desired. This is in agreement with the general rule of thumb for choosing the impedance parameters such that the manipulator impedance is proportional to the environment admittance in order to optimize the weighted velocity-force error [35]. With the proposed control law, the different task characteristics for grasping and tracking can be taken into consideration by choosing high local stiffness $\boldsymbol{K}$ and negative weights $\boldsymbol{K}^{s}$ for the synchronization. In order to avoid oscillations due to the second-order dynamics, while avoiding over-damped behavior resulting in a slower response time of the system, typically critically damped behavior is desired. For the impedance dynamics (4) this can be achieved with a suitable choice of the damping matrix as

$$
\boldsymbol{D}=2(\boldsymbol{M} \boldsymbol{K})^{\frac{1}{2}}
$$

With the proposed control law, the weights $\boldsymbol{K}^{s}$ have to be taken into account. This implies that by lowering the effective relative stiffness $\boldsymbol{K}_{e}$ with negative weights $\boldsymbol{K}^{s}$, the damping has to be reduced by choosing appropriate weights $\boldsymbol{D}^{s}$. Additionally, in a cooperative manipulation task not only the impedance parameters but also the object properties should be taken into account for the damping design. Consider again the object model (44). The effective relative stiffness at the end-effectors is increased to $\boldsymbol{K}_{e}+\left(\boldsymbol{N}^{\dagger}\right)^{T} \boldsymbol{K}_{L} \boldsymbol{N}^{\dagger}$, while the cooperative stiffness $\boldsymbol{K}_{c}$ is not affected, which should be considered in the damping design by increasing the relative damping $\boldsymbol{D}_{e}$ to a critically damping according to (49). As a second object property, the object inertia should be considered as motivated in the following.

Example 2: Assume a rigid object modeled as a simple point mass $M_{o} \in \mathbb{R}^{3 \times 3}$, where the center of mass is located at the geometrical center of the grasping points. Since the object is rigid, the resulting forces at the center of mass are obtained as the sum of the forces at the grasping points and thus

$$
\boldsymbol{h}_{c}^{o}=\boldsymbol{M}_{o} \ddot{\boldsymbol{x}}_{c} .
$$

In this case, the effective inertia for the cooperative task $\boldsymbol{M}_{c}+\boldsymbol{M}_{o}$ is increased and thus the damping $\boldsymbol{D}_{c}$ for the cooperative behavior should be increased towards the critical damping according to (49) in order to achieve a smooth transition.
Finally, the systems ability to reject disturbances has to be considered when choosing the impedance parameters. As apparent from Corollary 1 , the effect of the disturbance $\tilde{\boldsymbol{h}}$ can be reduced by increasing the damping and stiffness parameters $\boldsymbol{K}_{a}, \boldsymbol{D}_{a}$. However, recall that, while for the cooperative behavior high stiffness is desired, for the relative behavior low stiffness is desired to deal with model uncertainties. As a result, increasing both $\boldsymbol{K}_{a}$ and $\boldsymbol{D}_{a}$ results in better disturbance attenuation but negatively impacts the systems in regarding relative force tracking and convergence time.

In conclusion, we can see that the communication between agents allows us to shape the behaviors in the cooperative and relative sub-spaces independently. As a consequence, the behaviors can be tuned in order to meet the different performance requirements of the trajectory tracking and grasp maintenance task. Without the synchronization term (19), the parameters for the respective behaviors are coupled and defined via the impedance parameters $\boldsymbol{M}, \boldsymbol{D}, \boldsymbol{K}$ in (4) and an independent design is not possible.

\section{E. Effect of Communication Topology}

The connectivity between the agents, characterized by the reduced Laplacian $\boldsymbol{L}^{*}$, plays an important role for the error synchronization. Note that, opposed to typical consensus or formation control problems, in our case the connectivity does not only influence the convergence speed but more importantly the grasping force via the modified effective stiffness $\boldsymbol{K}_{e}$ and damping $\boldsymbol{D}_{e}$. For the sake of exposition, we will make some simplifications for the following discussion. First, we assume isotropic homogeneous parameters $\boldsymbol{K}_{k}^{s}=\kappa^{s} \boldsymbol{I}_{3}$ for all edges $k \in \mathcal{E}$ and $\boldsymbol{K}_{i}=\kappa \boldsymbol{I}_{3}$ for all agents $i$, where $\kappa^{s}, \kappa \in \mathbb{R}$. This allows us to write

$$
\boldsymbol{L}_{K}=\kappa^{s} \boldsymbol{L}^{*} \otimes \boldsymbol{I}_{3}, \quad \boldsymbol{K}=\kappa \boldsymbol{I}_{N} \otimes \boldsymbol{I}_{3} .
$$

Due to the properties of the Kronecker product, above equations are equal and decoupled for all spatial dimensions, and we focus the discussion on the one dimensional case as

$$
\boldsymbol{L}_{K}=\kappa^{s} \boldsymbol{L}^{*}, \quad \boldsymbol{K}=\kappa \boldsymbol{I}_{N} .
$$

Finally, in the following we only discuss the effect of the communication topology on the effective stiffness $\boldsymbol{K}_{e}$, but it should be noted that the results hold true for the effective damping $\boldsymbol{D}_{e}$ as well. As previously stated uncertainties in the object properties can lead to undesired interaction forces, which are amplified by the effective end-effector stiffness $\boldsymbol{K}_{e}$. Recall that $\boldsymbol{K}_{e}=\left(\boldsymbol{N}^{\dagger}\right)^{T}\left(\boldsymbol{K}+\boldsymbol{L}_{K}\right) \boldsymbol{N}^{\dagger}$. The spectrum of $\boldsymbol{L}_{K}$ is given by the eigenvalues of $\boldsymbol{L}^{*}$ multiplied by $\kappa^{s}$, which implies that the stiffness along the direction of the $i$ th eigenvector of $\boldsymbol{L}^{*}$ is modified as $\kappa+\kappa^{s} \lambda_{i}\left(\boldsymbol{L}^{*}\right)$. As a result, the eigenvalues and eigenvectors of $\boldsymbol{L}^{*}$ give important insights into the resulting relative stiffness.

Example 3: We illustrate the effect of the communication topology with the three different graphs in Fig. 1 in a simulation. Four agents modeled by (4), with $\boldsymbol{M}=$ $\boldsymbol{I}_{3 N \times 3 N}, \boldsymbol{D}=20 \boldsymbol{I}_{3 N \times 3 N}, \boldsymbol{K}=100 \boldsymbol{I}_{3 N \times 3 N}$ cooperatively manipulate a rigid object. The synchronization input (19) is used with $\boldsymbol{K}^{s}=-20 \boldsymbol{I}_{3 N \times 3 N}$ and $\boldsymbol{D}^{s}=\mathbf{0}_{|\mathcal{E}| \times|\mathcal{E}|}$. The initial 


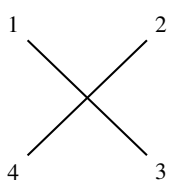

(a) Cross

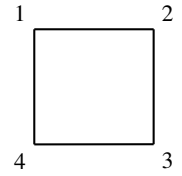

(b) Circle

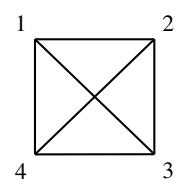

(c) Complete
Fig. 1. Different examples for the communication topology.

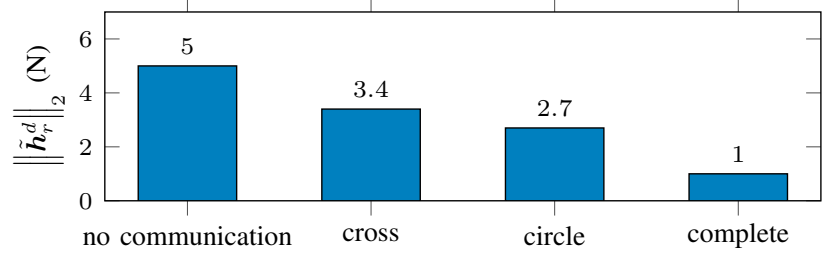

Fig. 2. Relative force error $\left\|\tilde{\boldsymbol{h}}_{r}^{d}\right\|_{2}$ for the case where no communication is present and where communication is present with a cross, circle and complete topology, respectively.

positions of the agents define a square around the object and the desired force is given as $10 \mathrm{~N}$ towards the object center. The set-point for the desired relative behavior is obtained by by letting $\boldsymbol{K}_{L, r} \rightarrow \infty$ in (48). However, we assume that the object geometry $\boldsymbol{r}$ is not precisely known and approximated by $\hat{\boldsymbol{r}}$, resulting in kinematic uncertainties $\tilde{\boldsymbol{r}}=\boldsymbol{r}-\hat{\boldsymbol{r}}=0.01 \mathbf{1}_{3(N-1)}$. The effect of the communication topology will be evaluated with the relative force error $\left\|\tilde{\boldsymbol{h}}_{l}^{d}\right\|_{2}=\left\|\boldsymbol{h}_{r}^{d}-\boldsymbol{h}_{r}\right\|_{2}$, where an index $l \in\{n, c r, c i, c o\}$ corresponds to the results with no communication and communication with cross, circle and complete graph, respectively. It should be noted that independent of the communication topology, $\boldsymbol{L}^{*}$ has at least one zero eigenvalue corresponding to the cooperative behavior and the following analysis focuses only on the three highest eigenvalues, characterizing the relative behavior. As depicted in Fig. 2, the relative force error is the highest in the case where no communication is present. While with the crossgraph Fig. 1 a communication amongst agents is present, the graph is not connected and we obtain a second zero eigenvalue $\lambda_{2}\left(\boldsymbol{L}^{*}\right)=0$, corresponding to the missing connections between the two sub-graphs. This implies that the relative stiffness can only be modified within the individual subgraphs, resulting in the highest absolute force errors for the three communication topologies. In contrast, the circle-graph in Fig. 1. b is connected and the relative stiffness along all directions can be effectively reduced. However, since the graph has missing edges between some agents, the eigenvalues of $\boldsymbol{L}^{*}$ are given as $\lambda_{i}\left(\boldsymbol{L}^{*}\right)=[0,2,2,4]$. The two eigenvalues $\lambda_{2}=\lambda_{3}=2$ correspond to the relative behavior between the not directly connected agents, while $\lambda_{4}=4$ corresponds to the relative behavior of the directly connected agents. This implies that the relative effective stiffness between the directly connected agents can be set lower than the relative stiffness of the agents where no direct path exists, resulting in lower errors than for the cross graph. Finally, for the complete-graph Fig. 1 c the eigenvalues of $\boldsymbol{L}^{*}$ are given as $\lambda_{2}=\lambda_{3}=\lambda_{4}=4$, resulting in an equally distributed relative stiffness between all

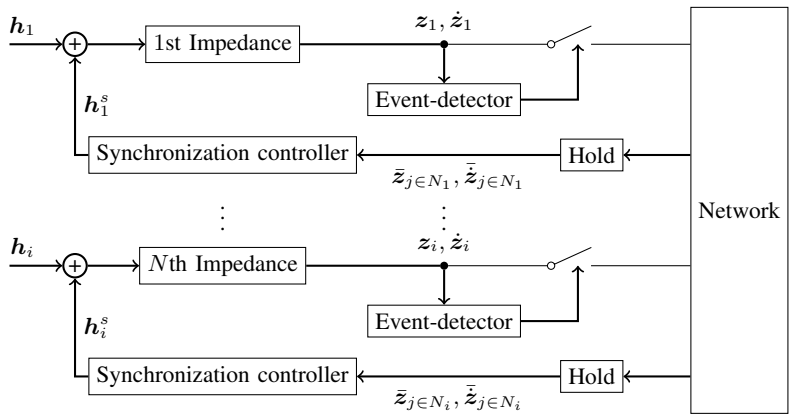

Fig. 3. Scheme of the control framework. The control, synchronization and event-detection all work only based on local information. The "Hold" block updates the local sample whenever an event is triggered.

agents. This can be seen in the relative force errors, which are the lowest for the complete-graph.

In conclusion, the effective relative stiffness does not only depend on the parameter $\kappa^{s}$ but also on the connectivity of the underlying graph; increasing the connectivity between agents leads to similar relative stiffnesses between individual agents and therefore a more balanced force distribution along all directions of the object.

\section{EVENT-TRIGGERED COMMUNICATION}

With the control law developed in the last section, agents exchange their signals $\boldsymbol{z}_{i}, \dot{\boldsymbol{z}}_{i}$ via a communication network. Depending on the number of agents, this can lead to a variety of negative effects like delay via network congestion and packet dropouts, which become more serious if all agents transmit continuously. In order to reduce the communication rate between agents, we propose an event-triggered communication strategy. This means that each agent $i$ transmits its signals $\boldsymbol{z}_{i}, \dot{\boldsymbol{z}}_{i}$ only at certain time instances $t_{k_{i}}$ with $k_{i}=$ $0,1, \ldots$ to its neighbors, which hold the information until it is updated again. We will derive a state-dependent triggering condition $f(\boldsymbol{z}, \dot{\boldsymbol{z}})$ such that the triggering instances $t_{k_{i}}$ are chosen whenever a predefined error $\left\|\boldsymbol{e}_{i}\right\|_{2}$ reaches a certain threshold as

$$
t_{k_{i}+1}=\inf \left\{t>t_{k_{i}} \mid\left\|\boldsymbol{e}_{i}\right\|_{2}=f\left(\boldsymbol{z}_{i}, \dot{\boldsymbol{z}}_{i}\right)\right\} .
$$

Note that in between two triggering instances agent $i$ has no information about the current state of agent $j$ and therefore only local information $\dot{z}_{i}, z_{i}$ can be incorporated to decide the next trigger instance, resulting in a decentralized eventtriggering scheme. Often times this is circumvented by assuming additional sensors at the $i$ th agent, which can measure the distance $\boldsymbol{z}_{j}-\boldsymbol{z}_{i}$ to neighboring agents (e.g. in [21], [27]). We will refrain from that and present a triggering mechanism, which only depends on local information, without the necessity of additional sensors. Also note that for such a decentralized scheme, asynchronous triggering is natural, i.e. the triggering instants at agent $i$ and $j$ are different in general. This means that the decision of transmission is done by the respective agent itself and the corresponding states $\dot{\boldsymbol{z}}_{i}, \boldsymbol{z}_{i}$ are transmitted to all of the neighbors at time $t_{k_{i}}$. Finally, avoidance of Zeno behavior, where an event is triggered infinitely often 
in a finite time interval, is a common problem in eventtriggered systems. In the following we will present a Zeno free triggering condition under which the system remains stable.

\section{A. Triggering Condition}

By defining

$$
\left.\begin{array}{l}
\overline{\boldsymbol{z}}_{i}(t)=\boldsymbol{z}_{i}\left(t_{k_{i}}\right) \\
\dot{\boldsymbol{z}}_{i}(t)=\dot{\boldsymbol{z}}_{i}\left(t_{k_{i}}\right)
\end{array}\right\} \forall t \in\left[t_{k_{i}}, t_{k_{i}+1}\right), k_{i} \in \mathbb{N}
$$

as the last transmitted signal $\boldsymbol{z}_{i}$ and $\dot{\boldsymbol{z}}_{i}$ of agent $i$, the synchronization input in (18) changes to

$$
\overline{\boldsymbol{h}}_{i}^{s}=\sum_{j \in N_{i}} \boldsymbol{D}_{k}^{s}\left(\overline{\dot{\boldsymbol{z}}}_{j}-\overline{\dot{\boldsymbol{z}}}_{i}\right)+\sum_{j \in N_{i}} \boldsymbol{K}_{k}^{s}\left(\overline{\boldsymbol{z}}_{j}-\overline{\boldsymbol{z}}_{i}\right)
$$

which can be written in compact form as

$$
\overline{\boldsymbol{h}}^{s}=-\boldsymbol{L}_{D} \overline{\dot{z}}-\boldsymbol{L}_{K} \overline{\boldsymbol{z}},
$$

and the closed loop error dynamics are obtained as

$$
M \ddot{z}+D \dot{z}+K z+L_{D} \overline{\dot{z}}+L_{K} \bar{z}=h+\tilde{h} .
$$

The resulting block diagram of the event-triggered control scheme can be found in Fig. 3 . Define the following triggering errors as

$$
\begin{aligned}
\tilde{\boldsymbol{z}}_{i} & =\overline{\boldsymbol{z}}_{i}-\boldsymbol{z}_{i} \\
\tilde{\dot{\boldsymbol{z}}}_{i} & =\overline{\dot{\boldsymbol{z}}}_{i}-\dot{\boldsymbol{z}}_{i} \\
\boldsymbol{e}_{i} & =\operatorname{vec}_{j \in N_{i}}\left(\boldsymbol{e}_{i j}\right),
\end{aligned}
$$

where $\boldsymbol{e}_{i j}=\boldsymbol{D}_{i j}^{s} \tilde{\dot{z}}_{i}+\boldsymbol{K}_{i j}^{s} \tilde{z}_{i}$ and $\operatorname{vec}_{j \in N_{i}}\left(\boldsymbol{e}_{i j}\right)$ denotes the column vector obtained by stacking all $\boldsymbol{e}_{i j}$, for which $j \in N_{i}$. We are now ready to formulate the triggering law as

$$
t_{k_{i}+1}=\inf \left\{t>t_{k_{i}} \mid\left\|\boldsymbol{e}_{i}(t)\right\|_{2}^{2}=\max \left\{\mu\left\|\dot{\boldsymbol{z}}_{i}(t)\right\|_{2}^{2}, \omega_{i}(t)\right\}\right\},
$$

with

$$
\mu=\delta \frac{\lambda_{d}^{2}}{4\left|N_{i}\right|},
$$

where $0<\delta<1$ and $\omega_{i}(t)$ is the solution of the initial value problem

$$
\dot{\omega}_{i}=-\nu \omega_{i}, \quad \omega_{i}\left(t_{0}\right)=\omega_{i 0}>0,
$$

with a scalar $\nu>0$. It is straightforward to show that $\omega_{i}(t)>$ $0, \forall 0 \leq t<\infty$ and $\lim _{t \rightarrow \infty} \omega_{i}(t)=0$.

\section{B. Triggering as Disturbance}

The general goal for impedance control schemes is not to directly control the force or motion errors but to impose a desired dynamical behavior on the system. In order to characterize deviations from this desired behavior, we analyze the system with event-triggered synchronization input (56) as a disturbed version of the system with time continuous synchronization input (19), where the trigger-induced disturbance is given as

$$
\tilde{\boldsymbol{h}}^{s}=\overline{\boldsymbol{h}}^{s}-\boldsymbol{h}^{s}=-\boldsymbol{L}_{D} \tilde{\dot{\boldsymbol{z}}}-\boldsymbol{L}_{K} \tilde{\boldsymbol{z}} .
$$

Before stating the corresponding stability and performance results, we provide the following upper bound on the triggerinduced disturbance.
Lemma 2: If the triggering instances $t_{k_{i}+1}$ are locally chosen according to 61 then the trigger-induced disturbance $\tilde{\boldsymbol{h}}^{s}$ is upper bounded as

$$
\left\|\tilde{\boldsymbol{h}}^{s}\right\|_{2}^{2} \leq \max \left\{\delta \lambda_{d}^{2}\|\dot{\boldsymbol{z}}\|_{2}^{2}, \beta\right\},
$$

where $\beta=\sum_{i=1}^{N} 4\left|N_{i}\right| \omega_{i}$.

Proof: With Young's inequality, we can write

$$
\begin{aligned}
\left\|\tilde{\boldsymbol{h}}^{s}\right\|_{2}^{2} & =\sum_{i=1}^{N}\left(\tilde{\boldsymbol{h}}_{i}^{s}\right)^{T} \sum_{j \in N_{i}}\left(\boldsymbol{e}_{i j}-\boldsymbol{e}_{j i}\right) \\
& \leq \sum_{i=1}^{N} \sum_{j \in N_{i}}\left[\frac{1}{2\left|N_{i}\right|}\left\|\tilde{\boldsymbol{h}}_{i}^{s}\right\|_{2}^{2}+\left|N_{i}\right|\left(\left\|\boldsymbol{e}_{i j}\right\|_{2}^{2}+\left\|\boldsymbol{e}_{j i}\right\|_{2}^{2}\right)\right] .
\end{aligned}
$$

Note that by Assumption 2 the graph is balanced and as a result above inequality can be further simplified as

$$
\left\|\tilde{\boldsymbol{h}}^{s}\right\|_{2}^{2} \leq \sum_{i=1}^{N} \sum_{j \in N_{i}}\left[\frac{1}{2\left|N_{i}\right|}\left\|\tilde{\boldsymbol{h}}_{i}^{s}\right\|_{2}^{2}+2\left|N_{i}\right|\left\|\boldsymbol{e}_{i j}\right\|_{2}^{2}\right] .
$$

Solving for $\left\|\tilde{\boldsymbol{h}}^{s}\right\|_{2}^{2}$ results in

$$
\left\|\tilde{\boldsymbol{h}}^{s}\right\|_{2}^{2} \leq \sum_{i=1}^{N} 4\left|N_{i}\right|\left\|\boldsymbol{e}_{i}\right\|_{2}^{2} .
$$

Considering that the triggering condition always ensures that $\left\|\boldsymbol{e}_{i}\right\|_{2}^{2} \leq \max \left\{\mu\left\|\dot{\boldsymbol{z}}_{i}\right\|_{2}^{2}, \omega_{i}\right\}$ completes the proof.

With this result we can immediately obtain performance criteria for the event-triggered system similar to those of the time-continuous system presented in Corollary 1 .

Corollary 2: Consider multiple impedance-controlled manipulators with error dynamics (4) cooperatively manipulating an object, where the additional control input $\boldsymbol{h}^{s}$ is obtained via the event-triggered control law (56). Let further Assumptions 122 hold, the weight matrices be chosen to fulfill (29), and the triggering instances $t_{k_{i}+1}$ be locally chosen according to 61]. Then the coupled manipulator dynamics (57) are $\mathcal{L}_{2}$ stable with

$$
\begin{aligned}
\|\dot{\boldsymbol{z}}\|_{\mathcal{L}_{2}} & \leq \frac{\gamma_{1}}{1-\sqrt{\delta}}\|\tilde{\boldsymbol{h}}\|_{\mathcal{L}_{2}}+\bar{\alpha}_{\dot{\boldsymbol{z}}} \\
\|\boldsymbol{z}\|_{\mathcal{L}_{2}} & \leq \gamma_{2}\|\boldsymbol{h}\|_{\mathcal{L}_{2}}+\frac{\gamma_{3}}{1-\sqrt{\delta}}\|\tilde{\boldsymbol{h}}\|_{\mathcal{L}_{2}}+\bar{\alpha}_{\boldsymbol{z}},
\end{aligned}
$$

where $\bar{\alpha}_{\dot{\boldsymbol{z}}}, \bar{\alpha}_{\boldsymbol{z}}$ are positive scalars.

Proof: The result is immediately obtained by substituting (65) into 36) and (37).

Note that the $\mathcal{L}_{2}$ gain of the disturbance input $\tilde{\boldsymbol{h}}$ is higher for the event-triggered case compared to the time-continuous counterpart. As a consequence, a given disturbance $\tilde{h}$ can lead to higher errors for the event-triggered system compared to its time-continuous counterpart, resulting in deteriorated performance of the system. This is to be expected since the triggering itself can be seen as a disturbance acting on the system and therefore less energy is available for stabilization of the system. Although $\delta$ can be chosen arbitrary close to zero, this leads to a lower threshold for the trigger condition (61) and can thus lead to more frequent triggering. This reveals a trade- 
off between reducing the communication between agents and the systems ability to reject disturbances. It should be noted that synchronization and as a result the triggering only affects the relative behavior. The cooperative tracking is completely unaffected by the communication strategy. In addition, since $\boldsymbol{e} \rightarrow \mathbf{0}$, in the case of $\tilde{\boldsymbol{h}}=\mathbf{0}$, only the transient behavior is affected by the triggering.

\section{Passivity}

We are now ready to state the main passivity result for the distributed control law with event-triggered communication.

Theorem 2: Consider multiple impedance-controlled manipulators with error dynamics (4) cooperatively manipulating an object, where the additional control input $\boldsymbol{h}^{s}$ is obtained via the event-triggered control law (56). Let further Assumption 2 hold, the weight matrices be chosen to fulfill (29), and the triggering instances $t_{k_{i}+1}$ be locally chosen according to 61. Then the system 57) is output-strictly-passive with inputoutput pair $[\boldsymbol{u}, \dot{\boldsymbol{z}}]$ and passivity index

$$
\rho^{*}=(1-\sqrt{\delta}) \rho .
$$

If in addition no disturbance is present, i.e. $\tilde{\boldsymbol{h}}=\mathbf{0}_{3 \mathrm{~N}}$ and Assumption 11 is fulfilled, then the system is stable with $\lim _{t \rightarrow \infty} \dot{\boldsymbol{z}}, \boldsymbol{e}=\mathbf{0}$ and inter-event times are lower bounded by

$$
t_{k_{i}+1}-t_{k_{i}} \geq \frac{1}{\tau} \max \left\{\sqrt{\mu}\left\|\dot{z}_{i}\right\|_{2}, \sqrt{\omega_{i}}\right\},
$$

where $\tau>0$ is a positive scalar.

Proof: Consider the Lyapunov candidate

$$
W=V_{0}+\frac{\beta^{2}}{2 \nu \sqrt{\delta} \lambda_{d}}
$$

Taking the derivative along the solution of (57) one obtains

$$
\begin{aligned}
\dot{W} & =\dot{\boldsymbol{z}}^{T}\left(\boldsymbol{u}+\tilde{\boldsymbol{h}}^{s}\right)-\dot{\boldsymbol{z}}^{T} \boldsymbol{D} \dot{\boldsymbol{z}}-\frac{1}{\sqrt{\delta} \lambda_{d}} \beta^{2} \\
& \leq \dot{\boldsymbol{z}}^{T} \boldsymbol{u}-\lambda_{d}\|\dot{\boldsymbol{z}}\|_{2}^{2}+\|\dot{\boldsymbol{z}}\|_{2} \max \left\{\sqrt{\delta} \lambda_{d}\|\dot{\boldsymbol{z}}\|_{2}, \beta\right\}-\frac{1}{\sqrt{\delta} \lambda_{d}} \beta^{2}
\end{aligned}
$$

$$
\leq \dot{\boldsymbol{z}}^{T} \boldsymbol{u}-(1-\sqrt{\delta}) \lambda_{d}\|\dot{\boldsymbol{z}}\|_{2}^{2},
$$

from which the OSP property can be concluded. In the case that $\tilde{\boldsymbol{h}}=\mathbf{0}_{3 N}$ and Assumption 1 is fulfilled, we obtain

$$
\dot{W}+\dot{V}_{e}=-(1-\sqrt{\delta}) \lambda_{d}\|\dot{z}\|_{2}^{2},
$$

from which $\dot{z} \rightarrow \mathbf{0}$ and thus $\boldsymbol{e} \rightarrow \mathbf{0}$ can be concluded. In addition, $W$ is bounded, which implies that $\dot{\boldsymbol{z}}, \boldsymbol{z}$ and thus $\overline{\dot{z}}, \overline{\boldsymbol{z}}$ are bounded as well. Based on Assumption 1, $\boldsymbol{h}$ is bounded since $\dot{z}$ is bounded and by inspecting 57 boundedness of $\ddot{z}$ follows immediately. Considering the time interval $\left[t_{k_{i}}, t_{k_{i}+1}\right)$, the derivative of $\left\|e_{i}\right\|_{2}$ with respect to time is bounded as

$$
\frac{d}{d t}\left\|\boldsymbol{e}_{i}\right\|_{2} \leq\left\|\dot{e}_{i}\right\|_{2} \leq\left\|\boldsymbol{D}_{i}^{s} \ddot{z}_{i}\right\|_{2}+\left\|\boldsymbol{K}_{i}^{s} \dot{\boldsymbol{z}}_{i}\right\|_{2},
$$

where $\boldsymbol{D}_{i}^{s}=\left[\left(\boldsymbol{D}_{i 1}^{s}\right)^{T} \ldots\left(\boldsymbol{D}_{i\left|N_{i}\right|}^{s}\right)^{T}\right]^{T}$ and $\boldsymbol{K}_{i}^{s}=$ $\left[\left(\boldsymbol{K}_{i 1}^{s}\right)^{T} \ldots\left(\boldsymbol{K}_{i\left|N_{i}\right|}^{s}\right)^{T}\right]^{T}$. Since $\ddot{z}$ and $\dot{z}$ are bounded we can conclude that there exist a $\tau \geq 0$ such that

$$
\frac{d}{d t}\left\|\boldsymbol{e}_{i}\right\|_{2} \leq \tau
$$

Integrating both sides over $\left[t_{k_{i}}, t\right]$ for any $t \in\left[t_{k_{i}}, t_{k_{i}+1}\right)$ yields,

$$
\left\|e_{i}\right\|_{2} \leq \tau\left(t-t_{k_{i}}\right)
$$

Recall that an event is triggered at time $t_{k_{i}+1}$ if

$$
\left\|\boldsymbol{e}_{i}\right\|_{2}=\max \left\{\sqrt{\mu}\left\|\dot{\boldsymbol{z}}_{i}\right\|_{2}, \sqrt{\omega_{i}}\right\} \leq \tau\left(t_{k_{i}+1}-t_{k_{i}}\right),
$$

resulting in the bound 73 .

Remark 7: Note that the discussion in Remark 3 still holds since $\|\boldsymbol{e}\|_{2} \rightarrow \mathbf{0}_{3 N}$. Thus the event-triggered system has the same steady-state properties as the time-continuous one. Consequently, merely the transient behavior and the disturbance attenuation properties are influenced by the triggering.

The proposed triggering condition can be interpreted from an energetic point of view. Without the triggering, energy is dissipated by the system via the damping term $\lambda_{d} \dot{z}$ as stated in Theorem 1 . The triggering condition uses this excess of energy to render the system stable under the discontinuous triggerinduced errors $\tilde{\boldsymbol{z}}, \tilde{\dot{\boldsymbol{z}}}$. The proportion of the energy sacrificed for this stabilization process to the energy dissipated can be set with the triggering parameter $\delta$. As a result, the amount of communication between agents can be reduced while guaranteeing stability of the system. However, as mentioned previously, this comes with the cost of a lower passivity index $\rho^{*}$, negatively impacting the performance in terms of the resulting $\mathcal{L}_{2}$-gain of the system. In conclusion, there is a trade-off between the amount of communication between agents and the tracking capabilities of the system.

\section{Effect of the Communication Topology}

The communication topology, characterized by the graph Laplacian $\boldsymbol{L}^{*}$, has various effects on the behavior of the triggering and therefore on the performance. As observable from the triggering condition 61, a higher number of neighbors $\left|N_{i}\right|$ for agent $i$ leads to a lower threshold on the triggering error $e_{i}$. As a direct consequence, the lower bound on the inter-event times in (73) is reduced, which can lead to more frequent triggering. While in the previous discussion a lower triggering threshold also implies a reduced upper bound on the trigger-induced disturbance, this is not necessarily the case anymore when the threshold is lowered due to a high number of neighbors. By inspection of 65 it can be seen that $\beta$ includes the terms $\left|N_{i}\right|$ and can therefore negatively affect the upper-bound on the event-triggered disturbance $\left\|\tilde{\boldsymbol{h}}^{s}\right\|_{2}$. Even in the case that $\delta \lambda_{d}^{2}\|\dot{\boldsymbol{z}}\|_{2}^{2} \leq \beta$, no improvement of the bound can be obtained. This is to be expected since a denser topology introduces more transmissions and with that triggering has a higher negative impact. It should be noted that the resulting passivity index $\rho^{*}$ of the event-triggered system, and with that the systems robustness against disturbances $\tilde{\boldsymbol{h}}$, does not depend on the number of neighbors and is thus unaffected by the choice of the communication topology. We can thus conclude that the negative effects of the event-triggering in the sense of the number of events and the trigger-induced disturbance are 
amplified with an increasing number of neighbors per agent. However, recalling the results in Section III-E, generally a densely connected communication topology is desired in order to achieve an equally distributed impedance behavior. As a consequence, similar to the previous discussion, a trade-off between the desired impedance and triggering behavior has to be considered.

\section{E. Guidelines for Controller Design}

The general design for the impedance parameters $M, D, K$ and the parameters for the synchronization controller $\boldsymbol{D}^{s}, \boldsymbol{K}^{s}$ mainly follows the ideas discussed in Section III-D The main difference is that high synchronization parameters $\boldsymbol{D}^{s}$ and $\boldsymbol{K}^{s}$ lead to a higher triggering error $e$, which can result in more frequent triggering. If communication needs to be reduced then low parameters $\boldsymbol{D}^{s}$ and $\boldsymbol{K}^{s}$ are desirable, again highlighting the trade-off between the number of events and the resulting behavior. The triggering parameter $\delta$ directly shapes the triggering threshold (61); higher $\delta$ leads to a higher triggering threshold resulting in a lower number of events but increases the trigger-induced disturbance. The choice of parameters for designing 63 depends on the task characteristics. As an example, the decay rate $\nu$ and initial state $\omega_{i 0}$ should be chosen with the task duration in mind such to avoid dense triggering during the task, while not affecting the convergence time of the system by having high $\omega_{i}$ at the end of the task. In addition, the communication topology and all other parameters have to be considered. Generally, if an effect previously discussed is expected to lead to dense triggering this can be compensated with an appropriate design of the $\omega_{i}$ dynamics. However, doing so comes with the cost of worse performance in the relative sub-task.

\section{Simulations}

In this section the results are illustrated by simulating four manipulators cooperatively manipulating an object. The manipulators are implemented via (4), with (19) for the time-continuous and (56) for the event-triggered case. The effect of different communication topologies on the results are illustrated with the three basic communication graphs presented in Section III-E as well as the case of no communication. The contact with a flexible object is modeled via a spring as in (44). We assume that the object stiffness is unknown and apply the desired forces as feed-forward term. The synchronization parameters are chosen negative definite to bring the relative stiffness close to zero as mentioned in Section III-C. All the following results are obtained via MonteCarlo-Simulation over 50 measurements with randomized parameters $\boldsymbol{\theta}_{r} \in\left\{\boldsymbol{r}, \boldsymbol{h}_{r}^{d}, \boldsymbol{x}_{c}^{d}, \boldsymbol{K}_{o},\right\}$, the range of which can be found on the left in Table II and the parameters for the controller $\boldsymbol{\theta}_{c} \in\left\{\boldsymbol{M}, \boldsymbol{D}, \boldsymbol{K}, \boldsymbol{D}^{s}\right\}$ can be found on the right in Table II For the event-triggered controller, we chose $\delta=0.9$ and the dynamics in (63) with $\nu=-10$ and $\omega_{i 0}=0.5$, for all agents $i$. The sampling time for the simulation is $0.0005 \mathrm{~s}$ and the duration of each measurement is $1 \mathrm{~s}$.
TABLE I

RANGES OF RANDOMIZE PARAMETERS USED FOR ALL SIMULATIONS (LEFT) AND CONTROLLER PARAMETERS (RIGHT).

\begin{tabular}{c|c|ccc|c}
$\theta_{r}$ & $\min$ & $\max$ & & $\theta_{c}$ & value \\
\hline $\boldsymbol{r}$ & $0.25 \mathrm{~m}$ & $0.5 \mathrm{~m}$ & & $\boldsymbol{M}$ & $\boldsymbol{I}_{3 N}$ \\
$\boldsymbol{x}_{c}^{d}$ & $0.05 \mathrm{~m}$ & $0.1 \mathrm{~m}$ & & $\boldsymbol{D}$ & $63.2 \boldsymbol{I}_{3 N}$ \\
$\boldsymbol{h}_{r}^{d}$ & $5 \mathrm{~N}$ & $10 \mathrm{~N}$ & & $\boldsymbol{K}$ & $1000 \boldsymbol{I}_{3 N}$ \\
$\boldsymbol{K}_{o}$ & $150 \mathrm{Nm}^{-1}$ & $300 \mathrm{Nm}^{-1}$ & & $\boldsymbol{D}^{s}$ & $\mathbf{0}_{3|\mathcal{E}| \times 3|\mathcal{E}|}$
\end{tabular}

\section{A. Evaluation Criteria}

In the following an index $l \in\{n, c r, c i, c o\}$ for any signal, corresponds to the signal obtained by simulation where no communication is present $(n)$ or where communication is present with a cross $(\mathrm{cr})$, circle ( $\mathrm{ci}$ ) or complete ( $\mathrm{co})$ graph, respectively. As mentioned in the previous sections, employing the event-triggered controller, a compromise between the task performance and triggering performance has to be considered. The task performance relates to the achievement of the two goals of cooperative manipulation; cooperative tracking characterized by the cooperative tracking error $\left\|\boldsymbol{z}_{l}\right\|_{2}=\left\|\boldsymbol{z}_{c}\right\|_{2}$ and grasp maintenance characterized by the normalized relative force error defined as $\tilde{h}_{l}^{d}=\frac{\left\|\boldsymbol{h}_{r}-\boldsymbol{h}_{r}^{d}\right\|_{2}}{\left\|\boldsymbol{h}_{r}^{d}\right\|_{2}}$. The triggering performance is evaluated based on the average number of events and the minimum inter-event time $t^{\text {min }}$. All criteria are averaged over the 50 measurements except for the minimum inter-event time $t^{\text {min }}$, which corresponds to the minimum of all trials. As a result, in the case where dense triggering with $t^{\text {min }}<0.0005 \mathrm{~s}$ occurs, this information is not lost due to averaging and can be seen in the results with $t^{\text {min }}=0.0005 \mathrm{~s}$.

\section{B. Results}

The effect of the communication on the relative force error $\tilde{h}_{l}^{d}$ is depicted in the top of Fig. 4. The dashed lines correspond to the relative force error for the time-continuous controller, while the solid lines corresponds to the force error for the event-triggered controller. As expected, the relative force error decreases for lower synchronization parameters as well as denser communication topologies, independent of the sampling strategy. This confirms the previous discussion that low parameters are desired in order to achieve good performance in the force tracking task. Recall that the trigger induced disturbance converges to zero, resulting in neglectable force errors after $1 \mathrm{~s}$ of simulation. As a result, the difference in the depicted force error characterizes merely the transient phase, with maximum deviations obtained at the lowest simulated stiffness $\boldsymbol{K}_{s}=-250 \boldsymbol{I}_{3|\mathcal{E}|}$, implying slower convergence of the event-triggered controller. Fig. 5 confirms that the cooperative behavior is not affected by the error synchronization; neither the triggering nor the communication topology affects the cooperative tracking error, which asymptotically converges to zero independent of the type of controller used. As discussed in Section IV-D it can be seen in the middle and bottom plot of Fig. 4 that the triggering performance in terms of the average number of events as well as minimum interevent times decreases for denser communication topologies. Similar, by increasing the synchronization parameters $\left\|\boldsymbol{K}_{s}\right\|_{2}$ 

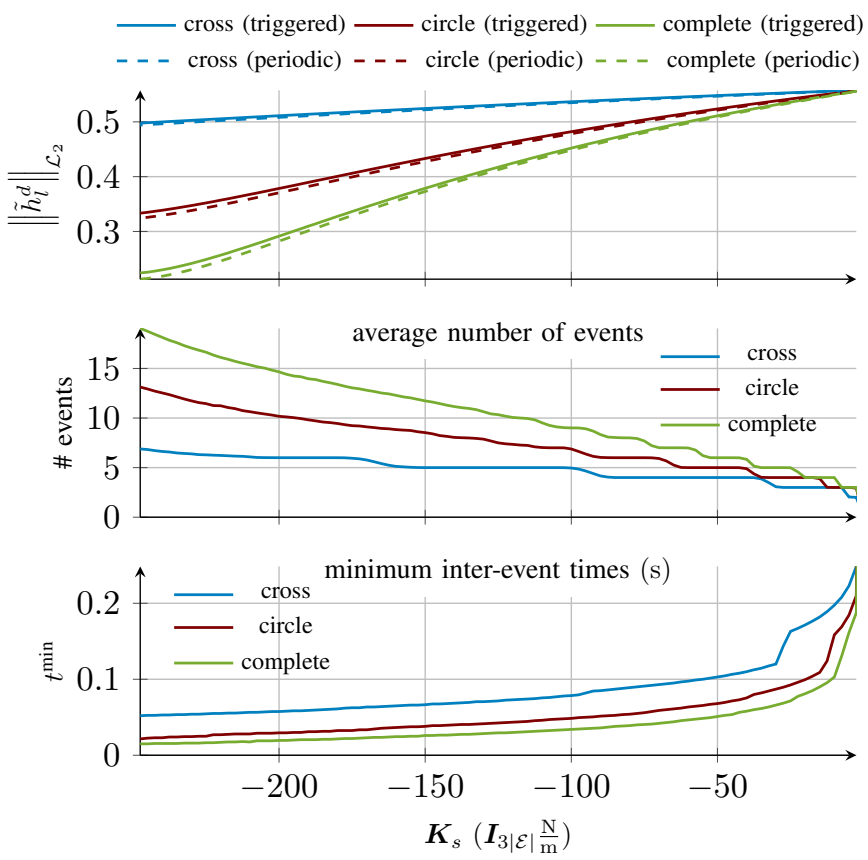

Fig. 4. From top to bottom: Normalized relative force errors $\left\|\tilde{\boldsymbol{h}}_{l}^{d}\right\|_{\mathcal{L}_{2}}$ of the time-continuous controller (dashed) compared to the event-triggered controller (solid). Average number of events and minimum inter-event times of the eventtriggered controller. All results are presented for the cross (blue), circle (red) and complete (green) communication graphs and different stiffness values.

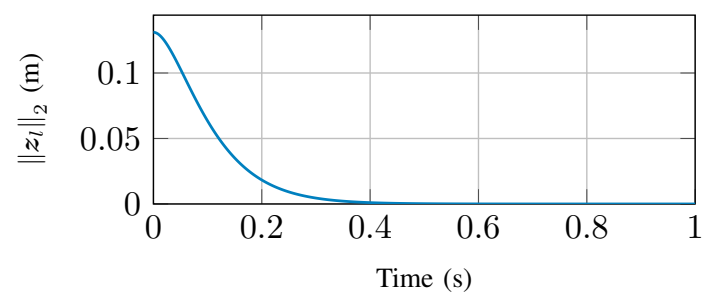

Fig. 5. Cooperative tracking error independent of the communication topology and sampling strategy.

the triggering performance decreases. This is to be expected, since increasing absolute parameters $\left\|\boldsymbol{K}_{s}\right\|_{2}$ leads to higher triggering errors $\|\boldsymbol{e}\|_{2}$. It should be noted that similar results regarding the triggering performance can be obtained for positive definite $\boldsymbol{K}_{s}$. As a conclusion, the triggering performance, in terms of the average number of events and minimum interevent times, decreases for denser communication topologies as well as high absolute parameters $\left\|\boldsymbol{K}_{s}\right\|_{2}$. Similar results can be obtained for $\left\|\boldsymbol{D}_{s}\right\|_{2}$, but are excluded due to space limitations. In total the average transmission rate can be reduced to less than $1 \%$, compared to a periodic sampling scheme with the same sampling rate of $0.0005 \mathrm{~s}$. In addition, a clear trade-off can be observed in terms of task performance and triggering performance. While low relative stiffness and with that high absolute synchronization parameters $\left\|\boldsymbol{K}_{s}\right\|_{2}$ and dense communication topologies are desired to achieve good task performance, the opposite is true for the triggering performance. Finally, since $t^{\text {min }}>0.0005$ s for all simulations, we can confirm that no Zeno behavior occurs.

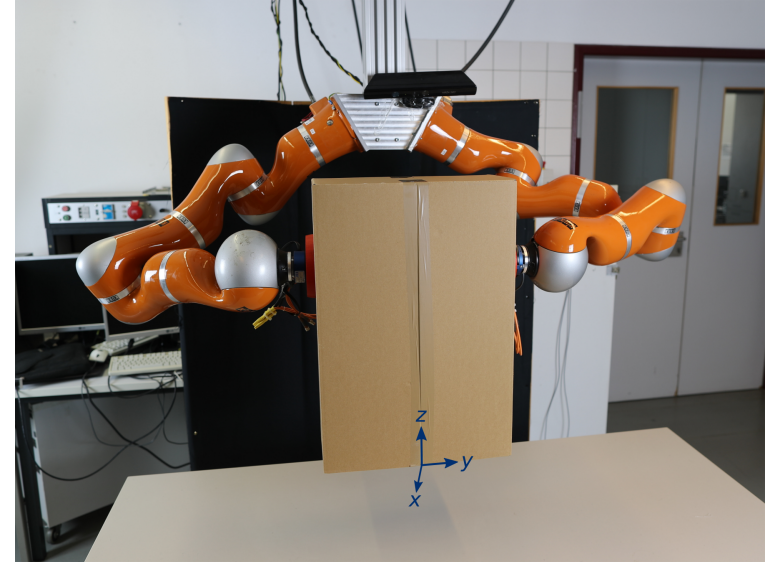

Fig. 6. Experimental setup consisting of two KUKA LWR 4+ with wrist mounted JR3 force-torque sensors. The world frame is marked in blue.

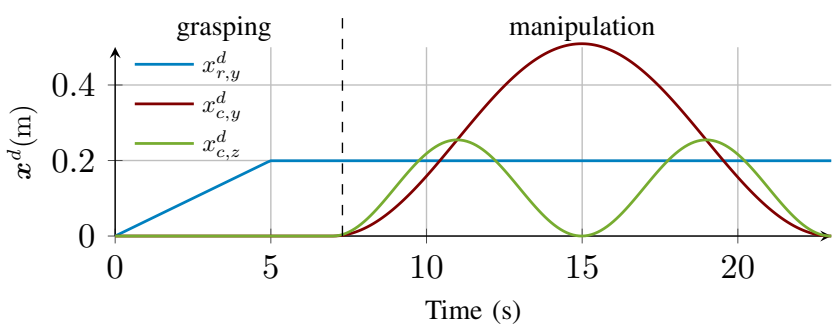

Fig. 7. Desired relative trajectory $x_{r, y}^{d}$ along the $y$-axis (blue) and desired cooperative trajectory $\boldsymbol{x}_{c}^{d}$ along the $y$ (red) and $z$-axis (green), respectively. All signals are with respect to the initial configuration.

\section{EXPERIMENTS}

In this section the performance of the proposed eventtriggered controller is experimentally evaluated. Similar as in the previous section, we first analyze the achievement of the two goals of cooperative manipulation and afterwards discuss the triggering-behavior. Finally, we analyze the effect of the object compliance on the triggering behavior.

\section{A. Experimental Setup}

The setup used for the experimental validation is shown in Fig. 6 As a proof of concept two 7 degree of freedom KUKA LWR 4+ manipulators cooperatively grasp and manipulate a cardboard-box. The individual impedance dynamics (4) are obtained by a position-based impedance approach with parameters $\boldsymbol{K}_{i}=500 \boldsymbol{I}_{3}, \boldsymbol{D}_{i}=150 \boldsymbol{I}_{3}, \boldsymbol{M}_{i}=10 \boldsymbol{I}_{3}$ and $\boldsymbol{K}_{i}^{s}=-100 \boldsymbol{I}_{3}, \boldsymbol{D}_{i}^{s}=\mathbf{0}_{3 \times 3}, \forall i \in\{1,2\}$ for the synchronization controller [56. We chose the parameters for the triggering condition 61) as $\delta=0.9, \eta=0.5$ and the dynamics in 63 with $\nu=-0.01$ and $\omega_{i 0}=0.1$. The frequency of the data acquisition and controller update is $1 \mathrm{kHz}$. The forces acting at the end-effectors are measured by wrist-mounted JR3 forcetorque sensors. The task is divided into two phases:

(i) The object is grasped by applying a relative desired velocity of $0.02 \mathrm{~ms}^{-1}$ until the desired set-point is reached, which is chosen by assuming a rigid object with $\boldsymbol{K}_{o} \rightarrow \infty$ in (48) such to achieve a grasping force of $60 N$. 


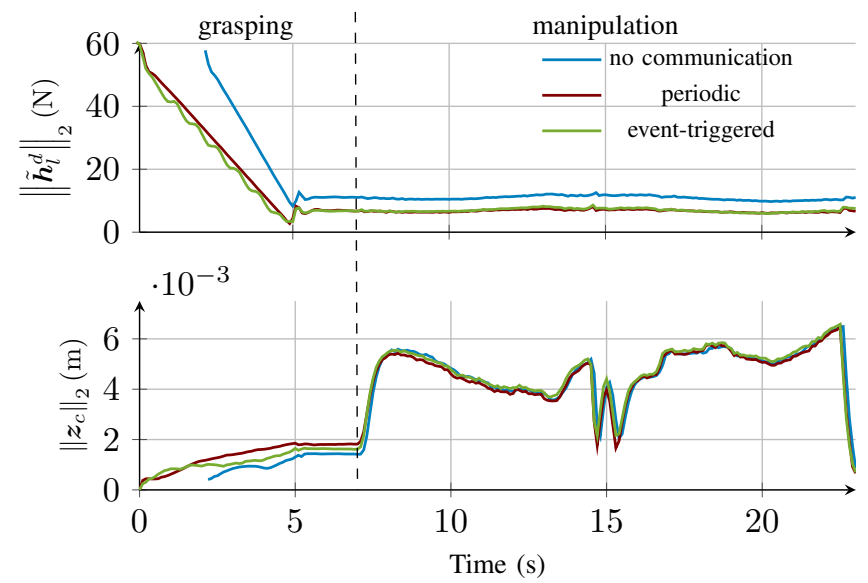

Fig. 8. Task performance during the grasping and manipulation phase. Relative force error $\left\|\tilde{\boldsymbol{h}}_{l}^{d}\right\|_{2}$ (top) and cooperative tracking error $\left\|\boldsymbol{z}_{c}\right\|_{2}$ (bottom) for the case where no communication is present ( $n$, blue), communication is present with an periodic ( $p$, red) and event-triggered $(t$, green) sampling strategy. In order to compare the results, the no communication case is shifted to align the manipulation phases.

(ii) After a waiting period of $3 s$, a cooperative desired velocity is given as a sine with amplitude of $0.1 \mathrm{~ms}^{-1}$ and frequency of $\frac{1}{16} \mathrm{~Hz}$ and $\frac{1}{8} \mathrm{~Hz}$ along the $y$ and $z$-axis respectively, which corresponds to a typical pick and place task as illustrated in Fig. 7 .

The relative forces are be obtained as $\boldsymbol{h}_{r}=\boldsymbol{h}_{1}-\boldsymbol{h}_{2}$ and we define the relative force error as $\tilde{\boldsymbol{h}}_{l}^{d}=\boldsymbol{h}_{r}-\boldsymbol{h}_{r}^{d}$, where an index $l \in\{n, p, t\}$ corresponds to the case where no communication $(n)$ is present or communication is present with a periodic $(p)$ or event-triggered $(t)$ strategy.

\section{B. Task Performance}

The norm of the relative forces for the event-triggered controller during the grasp and manipulation phases are depicted in Fig. 8 on the top. During grasping, both manipulators approach the object, leading to a decreasing relative force error. Once the object is grasped, the relative force error stays roughly constant at $\left\|\tilde{\boldsymbol{h}}_{t}^{d}\right\|_{2} \approx 7 \mathrm{~N}$. Compared to the relative force error without synchronization controller with $\left\|\tilde{\boldsymbol{h}}_{n}^{d}\right\|_{2} \approx 10.9 \mathrm{~N}$, the benefit of the communication can be observed with a reduction in the relative force error of approx $35 \%$. Between the event-triggered and periodic sampling approach no significant difference can be observed, with $\left\|\tilde{\boldsymbol{h}}_{p}^{d}\right\|_{2} \approx 6.8 \mathrm{~N}$. Merely in the grasping stage the effect of the triggering can be seen due to the steps in the relative force error. The norm of the cooperative tracking error is depicted in Fig. 8 on the top. It can be seen that during the grasping phase minor deviations occur. Those errors are due to a slight misalignment of the end-effectors with the object, as well as errors in the measured forces. At the start of the manipulation phase, the error suddenly increases due to the object gravity, which is considered a disturbance to the system. The peak at around $15 \mathrm{~s}$ comes from the contact of the object and environment. However, by comparing the cooperative tracking error $\left\|z_{c}\right\|_{2}$ for the controller without

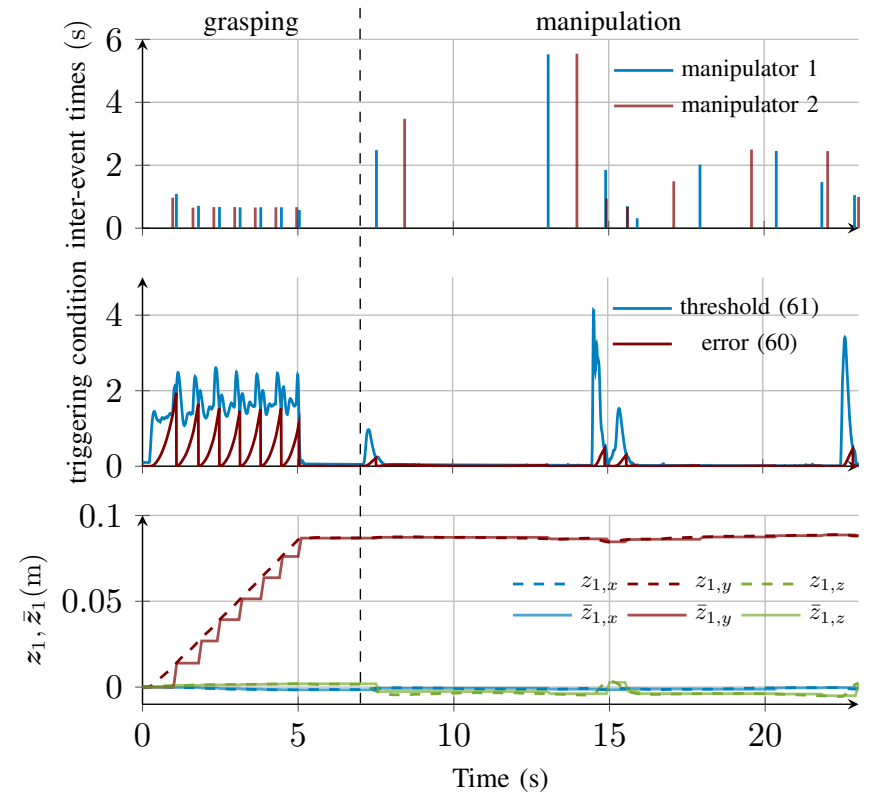

Fig. 9. Triggering behavior during the grasping and manipulation phase. From top to bottom: event-times for both manipulators, triggering threshold (blue) and error (red) for the first manipulator, event-triggered signal $\overline{\boldsymbol{z}}_{1}$ (solid) compared to the time-continuous counterpart $\boldsymbol{z}_{1}$ (dashed) for the first manipulator along the $x$ (blue), $y$ (red) and $z$-axis (green), respectively.

synchronization to the controller with synchronization and periodic and event-triggered communication strategy, it can be seen that all controllers perform similarly. Minor deviations in the observations can be explained by disturbances to the system and do not imply a systematic difference between the controllers.

\section{Triggering Performance}

The triggering behavior of the system is illustrated in Fig.9 During the manipulation phase, the position error $z$ increases fast since kinetic energy is converted to potential energy in the virtual spring, resulting in high error velocities $\dot{z}$. As a consequence, the triggering threshold in 61) increases and higher triggering-errors $\left\|\boldsymbol{e}_{i}\right\|_{2}$ are tolerated, resulting in an average of one transmission per second for both manipulators during the grasping phase. During the manipulation phase, only minor errors are introduced in the system since only the object dynamics act as an external force on the manipulators. Consequently, the tracking error stays relatively constant, resulting in a low threshold, which would lead to dense triggering. However, by choosing a suitable time-dependent threshold $\omega_{i}(t)$, minor triggering errors are tolerated and the transmission rate is lowered to 0.56 and 0.50 transmissions per second, for the left and right manipulator, respectively. It should be noted that $\omega_{i}(t)$ avoids unnecessary triggering in free motion, where the tracking error varies slowly. In contrast, during contact with the environment, the manipulators cannot track the desired motion and an event is triggered as it can be seen at the times around $15 \mathrm{~s}$ and $23 \mathrm{~s}$. In total with an average inter-event time of $1.43 \mathrm{~s}$ and $1.54 \mathrm{~s}$, leading to 16 and 15 transmissions for the left and right manipulator, the load on the 


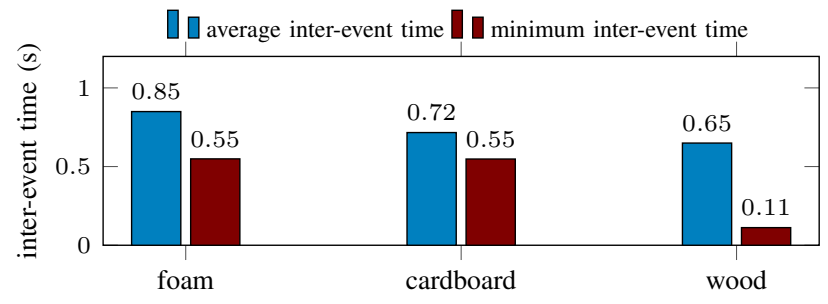

Fig. 10. Average (blue) and minimum (red) inter-event times for the foam block, the cardboard box and the wood block.
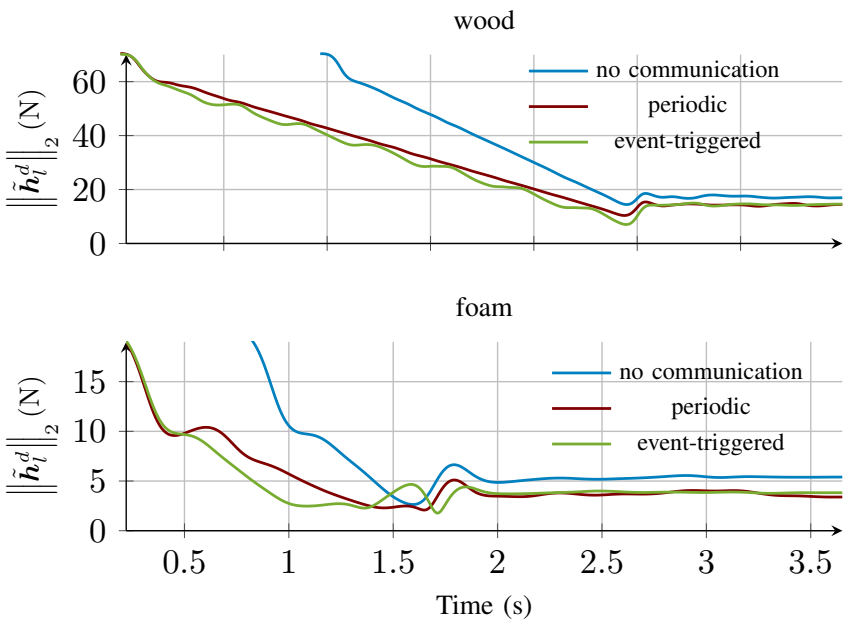

Fig. 11. Relative force error $\left\|\tilde{\boldsymbol{h}}_{l}^{d}\right\|_{2}$ during the grasping phase for the wood (top) and foam (bottom) block for the case where no communication is present ( $n$, blue), communication is present with an periodic ( $p$, red) and eventtriggered $(t$, green) sampling strategy. In order to compare the results, the no communication case is shifted to align the end of the grasping stage.

communication network is drastically reduced to less than $1 \%$ compared to a periodic sampling strategy. In addition, with minimum inter-event times of $0.32 \mathrm{~s}$ and $0.65 \mathrm{~s}$ for the left and right manipulator, the exclusion of Zeno behavior is confirmed.

\section{Influence of Object Compliance}

In order to see the influence of the object compliance on the proposed control scheme, in addition to the cardboard box, we perform experiments with a soft foam block as well as a rigid wood block. The control parameters are the same as in the first experiment, while the desired force is adjusted to $20 \mathrm{~N}$ for the foam and $70 \mathrm{~N}$ for the wood block to account for the different object masses. The triggering is analyzed based on the average number of events $t_{l}^{\text {avg }}$, where an index $l \in\{f, c, w\}$ corresponds to measurements with the foam, cardboard and wood object, respectively. We restrict the analysis to the grasping stage since triggering in the manipulations stage is mostly influenced by noise and the dynamics of $\omega$ as seen previously. In addition, since the duration for the grasping stage varies for the different objects, a performance criterion independent of the duration is desired, which is given by the average inter-event time instead of the total number of events. As observable from the results in Fig. 10, a more compliant object leads to less frequent triggering with $t_{f}^{\text {avg }}=0.85 \mathrm{~s}$, $t_{c}^{\text {avg }}=0.72 \mathrm{~s}$ and $t_{w}^{\text {avg }}=0.65 \mathrm{~s}$. In addition, it can be seen from the minimum inter-event times that no Zeno behavior occurs. From the force error during the grasping stage in Fig. 11 for the wood and foam block for the different communication strategies, it becomes evident that all the previous results still hold, in the sense that due to communication the relative force error is reduced by roughly $14 \%$ and $30 \%$ for the wood and foam block, respectively. More importantly, the system does not show any oscillatory behavior during all experiments conducted. This is especially remarkable for the wood block since interaction tasks with rigid environments typically pose severe requirements on the sampling time of the control law. We hypothesize that this is due to the fact that our proposed approach adapts the communication instances online in order to maintain the OSP characteristics of the time-continuous system, which are known to imply robustness.

\section{CONCLUSION}

In this article, a novel distributed cooperative manipulation approach is presented, which allows for more flexibility in the control design compared to a decentralized impedance scheme, while avoiding a vulnerable centralized control structure. The approach is analyzed by splitting the resulting dynamics into a cooperative and relative sub-space, directly corresponding to the tracking and grasp maintenance goals of cooperative manipulation. In order to reduce the load on the communication network, an event-triggered communication strategy is employed, maintaining the most important properties of output-strictly passive systems which are critical for interaction tasks. The effect of different communication topologies and controller parameters is analyzed in simulations and experimental evaluation on two KUKA LWR 4+ manipulators. It is demonstrated that the communication rate is drastically reduced, while maintaining a good performance with individually set impedance goals for the tracking and grasp maintenance tasks.

\section{REFERENCES}

[1] C. Smith, Y. Karayiannidis, L. Nalpantidis, X. Gratal, P. Qi, D. V. Dimarogonas, and D. Kragic, "Dual arm manipulationa survey," Robotics and Autonomous Systems, vol. 60, no. 10, pp. 1340 - 1353, 2012.

[2] S. Erhart and S. Hirche, "Model and analysis of the interaction dynamics in cooperative manipulation tasks," IEEE Transactions on Robotics, vol. 32, no. 3, pp. 672-683, June 2016.

[3] D. Sieber and S. Hirche, "Human-guided multirobot cooperative manipulation," IEEE Trans. on Control Systems Technology, pp. 1-18, 2018.

[4] G. Antonelli, F. Arrichiello, and S. Chiaverini, "The null-space-based behavioral control for autonomous robotic systems," Intelligent Service Robotics, vol. 1, no. 1, pp. 27-39, 2008.

[5] Y. Xu and J. P. Hespanha, "Communication logic design and analysis for networked control systems," in Current trends in nonlinear systems and control. Springer, 2006, pp. 495-514.

[6] J. G. Andrews, S. Buzzi, W. Choi, S. V. Hanly, A. Lozano, A. C. K. Soong, and J. C. Zhang, "What will $5 \mathrm{~g}$ be?" IEEE Journal on Selected Areas in Communications, vol. 32, no. 6, pp. 1065-1082, June 2014.

[7] L. Ding, Q. Han, X. Ge, and X. Zhang, "An overview of recent advances in event-triggered consensus of multiagent systems," IEEE Transactions on Cybernetics, vol. 48, no. 4, pp. 1110-1123, April 2018.

[8] O. Khatib, K. Yokoi, K. Chang, D. Ruspini, R. Holmberg, A. Casal, and A. Baader, "Force strategies for cooperative tasks in multiple mobile manipulation systems," Int. Symposium on Robotics Research, 1995.

[9] A. Marino, "Distributed adaptive control of networked cooperative mobile manipulators," IEEE Transactions on Control Systems Technology, vol. 26, no. 5, pp. 1646-1660, Sept 2018. 
[10] A. Petitti, A. Franchi, D. D. Paola, and A. Rizzo, "Decentralized motion control for cooperative manipulation with a team of networked mobile manipulators," in 2016 IEEE International Conference on Robotics and Automation (ICRA), May 2016, pp. 441-446.

[11] S. A. Schneider and R. H. Cannon, "Object impedance control for cooperative manipulation: theory and experimental results," IEEE Trans. on Robotics and Automation, vol. 8, no. 3, pp. 383-394, June 1992.

[12] R. C. Bonitz and T. C. Hsia, "Internal force-based impedance control for cooperating manipulators," IEEE Transactions on Robotics and Automation, vol. 12, no. 1, pp. 78-89, Feb 1996.

[13] F. Caccavale, P. Chiacchio, A. Marino, and L. Villani, "Six-dof impedance control of dual-arm cooperative manipulators," IEEE/ASME Transactions on Mechatronics, vol. 13, no. 5, pp. 576-586, Oct 2008.

[14] S. Musić, G. Salvietti, P. B. gen. Dohmann, F. Chinello, D. Prattichizzo, and S. Hirche, "Robot team teleoperation for cooperative manipulation using wearable haptics," in 2017 IEEE/RSJ International Conference on Intelligent Robots and Systems (IROS), Sept 2017, pp. 2556-2563.

[15] J. Szewczyk, F. Plumet, and P. Bidaud, "Planning and controlling cooperating robots through distributed impedance," Journal of Robotic Systems, vol. 19, no. 6, pp. 283-297, 2002.

[16] S. Erhart, D. Sieber, and S. Hirche, "An impedance-based control architecture for multi-robot cooperative dual-arm mobile manipulation," in 2013 IEEE/RSJ International Conference on Intelligent Robots and Systems, Nov 2013, pp. 315-322.

[17] W. P. M. H. Heemels, K. H. Johansson, and P. Tabuada, "An introduction to event-triggered and self-triggered control," in 2012 IEEE 51st IEEE Conference on Decision and Control (CDC), Dec 2012, pp. 3270-3285.

[18] Z.-P. Jiang and T.-F. Liu, "A survey of recent results in quantized and event-based nonlinear control," International Journal of Automation and Computing, vol. 12, no. 5, pp. 455-466, 2015.

[19] X. Li, X. Dong, Q. Li, and Z. Ren, "Decentralized event-triggered formation of linear multi-agent system," in 2017 13th IEEE International Conference on Control Automation (ICCA), July 2017, pp. 988-993.

[20] P. Yu, C. Fischione, and D. V. Dimarogonas, "Distributed event-triggered communication and control of linear multiagent systems under tactile communication," IEEE Transactions on Automatic Control, vol. 63 , no. 11, pp. 3979-3985, Nov 2018.

[21] D. V. Dimarogonas, E. Frazzoli, and K. H. Johansson, "Distributed event-triggered control for multi-agent systems," IEEE Transactions on Automatic Control, vol. 57, no. 5, pp. 1291-1297, May 2012.

[22] P. Tabuada, "Event-triggered real-time scheduling of stabilizing control tasks," IEEE Transactions on Automatic Control, vol. 52, no. 9, pp. 1680-1685, Sept 2007.

[23] N. Marchand, S. Durand, and J. F. G. Castellanos, "A general formula for event-based stabilization of nonlinear systems," IEEE Transactions on Automatic Control, vol. 58, no. 5, pp. 1332-1337, May 2013.

[24] J. F. Guerrero-Castellanos, N. Marchand, S. Durand, A. Vega-Alonzo, and J. J. Téllez-Guzmán, "Event-triggered attitude control for flying robots using an event approach based on the control," in 2015 International Conference on Event-based Control, Communication, and Signal Processing (EBCCSP), June 2015, pp. 1-8.

[25] B. Mu, H. Li, W. Li, and Y. Shi, "Consensus for multiple euler-lagrange dynamics with arbitrary sampling periods and event-triggered strategy," in Proceeding of the 11th World Congress on Intelligent Control and Automation, June 2014, pp. 2596-2601.

[26] N. Huang, Z. Duan, and Y. Zhao, "Distributed consensus for multiple euler-lagrange systems: An event-triggered approach," Science China Technological Sciences, vol. 59, no. 1, pp. 33-44, 2016.

[27] Q. Liu, M. Ye, J. Qin, and C. Yu, "Event-based leader-follower consensus for multiple euler-lagrange systems with parametric uncertainties," in 2016 IEEE 55th Conference on Decision and Control (CDC), Dec 2016, pp. 2240-2246.

[28] — , "Event-triggered algorithms for leader-follower consensus of networked euler-lagrange agents," IEEE Transactions on Systems, Man, and Cybernetics: Systems, pp. 1-13, 2018.

[29] Z. Sun, N. Huang, B. D. O. Anderson, and Z. Duan, "A new distributed zeno-free event-triggered algorithm for multi-agent consensus," in IEEE 55th Conference on Decision and Control (CDC), 2016, pp. 3444-3449.

[30] R. Ortega, J. A. L. Perez, P. J. Nicklasson, and H. J. Sira-Ramirez Passivity-based control of Euler-Lagrange systems: mechanical, electrical and electromechanical applications. Springer Science \& Business Media, 2013.

[31] F. Zhu, M. Xia, and P. J. Antsaklis, "On passivity analysis and passivation of event-triggered feedback systems using passivity indices," IEEE Trans. on Automatic Control, vol. 62, no. 3, pp. 1397-1402, 2017.

[32] H. Yu and P. J. Antsaklis, "Event-triggered output feedback control for networked control systems using passivity: Achieving $L_{2}$ stability in the presence of communication delays and signal quantization," Automatica, vol. 49, no. 1, pp. $30-38,2013$.

[33] A. Rahnama, M. Xia, and P. J. Antsaklis, "A QSR-dissipativity and passivity based analysis of event-triggered networked control systems," in 2016 IEEE 55th Conference on Decision and Control (CDC), Dec 2016, pp. 3072-3077.

[34] —, "Passivity-based design for event-triggered networked control systems," IEEE Transactions on Automatic Control, vol. 63, no. 9, pp. 2755-2770, Sept 2018

[35] N. Hogan, "Impedance control: An approach to manipulation: Part i-iii," Journal of dynamic systems, measurement, and control, vol. 107, no. 1, pp. 1-24, 1985

[36] D. A. Lawrence, "Impedance control stability properties in common implementations," in Proceedings. 1988 IEEE International Conference on Robotics and Automation, April 1988, pp. 1185-1190 vol.2.

[37] C. Ott, R. Mukherjee, and Y. Nakamura, "Unified impedance and admittance control," in 2010 IEEE International Conference on Robotics and Automation, May 2010, pp. 554-561.

[38] H. G. Tanner, K. J. Kyriakopoulos, and N. J. Krikelis, "Modeling of multiple mobile manipulators handling a common deformable object," Journal of Robotic Systems, vol. 15, no. 11, pp. 599-623, 1998.

[39] R. Ortega, A. Loria, R. Kelly, and L. Praly, "On passivity-based output feedback global stabilization of euler-lagrange systems," in 33rd IEEE Conference on Decision and Control, vol. 1, 1994, pp. 381-386 vol.1.

[40] D. Lee, "Distributed backstepping control of multiple thrust-propelled vehicles on a balanced graph," Automatica, vol. 48, no. 11, pp. 2971 2977, 2012.

[41] D. Lee and P. Y. Li, "Passive decomposition of mechanical systems with coordination requirement," IEEE Transactions on Automatic Control, vol. 58, no. 1, pp. 230-235, Jan 2013.

[42] H. Khalil, Nonlinear Systems. Prentice Hall, 2002.

[43] T. Hatanaka, Y. Igarashi, M. Fujita, and M. W. Spong, "Passivitybased pose synchronization in three dimensions," IEEE Transactions on Automatic Control, vol. 57, no. 2, pp. 360-375, Feb 2012.

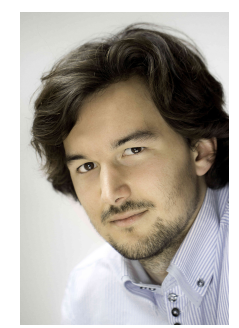

Pablo B. gen. Dohmann received his M.Sc. in Electrical and Computer Engineering from the Technical University of Munich, Germany in 2018. Since then he is pursuing the Dr.Ing. degree in Electrical and Computer Engineering at the Chair of Information-Oriented Control, Department of Electrical and Computer Engineering, Technical University of Munich. His research interest include multirobot systems, networked and distributed control.

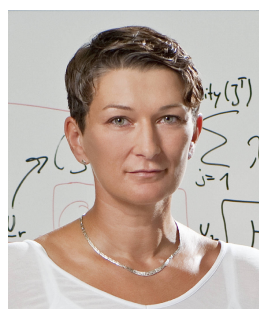

Sandra Hirche (M'03-SM'11) received the DiplomIngenieur degree in aeronautical engineering from Technical University Berlin, Germany, in 2002 and the Doktor-Ingenieur degree in electrical engineering from Technical University Munich, Germany, in 2005. From 2005 to 2007 she was awarded a Postdoc scholarship from the Japanese Society for the Promotion of Science at the Fujita Laboratory, Tokyo Institute of Technology, Tokyo, Japan. From 2008 to 2012 she has been an associate professor at Technical University Munich. Since 2013 she is TUM Liesel Beckmann Distinguished Professor and heads the Chair of Information-oriented Control in the Department of Electrical and Computer Engineering at Technical University Munich. Her main research interests include cooperative, distributed and networked control with applications in human-robot interaction, haptics, multi-robot systems, and general robotics. She has published more than 150 papers in international journals, books and refereed conferences. Dr. Hirche has served on the Editorial Boards of the IEEE Transactions on Control of Network Systems, IEEE Transactions on Control Systems Technology, and the IEEE Transactions on Haptics. She has received multiple awards such as the Rohde \& Schwarz Award for her $\mathrm{PhD}$ thesis in 2005, the IFAC World Congress Best Poster Award in 2005, Best Paper Awards at IEEE Worldhaptics and the ERC Starting Grant "Control based on Human Models" (2014-19). 